\title{
Heyting Mereology as a Framework for Spatial Reasoning
}

\author{
Thomas Mormann
}

$\underline{\text { Key Words: }}$ Mereology, Heyting algebras, co-Heyting algebras, Topology, Boundary, Interior Parts, Contact Relation, Representations.

Abstract: In this paper it is shown that Heyting and Co-Heyting mereological systems provide a convenient conceptual framework for spatial reasoning, in which spatial concepts such as connectedness, interior parts, (exterior) contact, and boundary can be defined in a natural and intuitively appealing way. This fact refutes the wide-spread contention that mereology cannot deal with the more advanced aspects of spatial reasoning and therefore has to be enhanced by further non-mereological concepts to overcome its congenital limitations. The allegedly unmereological concept of boundary is treated in detail and shown to be essentially affected by mereological considerations. More precisely, the concept of boundary turns out to be realizable in a variety of different mereologically grounded versions. In particular, every part $\mathrm{K}$ of a Heyting algebra $\mathrm{H}$ gives rise to a well-behaved K-relative boundary operator.

1. Introduction. In reasoning about space we employ a variety of concepts that come from different conceptual sources, e.g. geometry in the classical sense, topology, measure theory, information theory, cognitive psychology, physics. Although mereology may play an auxiliary role in spatial reasoning it has been considered as relevant only for its most elementary aspects. For more sophisticated spatial problems one has to rely on non-mereological concepts, or this is the generally accepted opinion:

[The conceptual limits of mereology] show up in any attempt to account for a number of important spatial concepts besides connectedness, such as the distinction between a completely interior part and a tangential part that is connected with the exterior, of the difference between an open entity and a closed one. All of these - and many others indeed - are relations that any theory concerned with the spatial structure of the world should supply and which cannot, however, be defined directly in terms of plain mereological primitives (Varzi 2007, 977-978). 
In this paper I want to show that this assessment is plainly wrong. ${ }^{1}$ Allegedly exclusively topological spatial concepts such as connectedness, the difference between tangential and nontangential (interior) parts, and a variety of different concepts of boundary can be formulated „,in terms of plain mereological concepts“ (ibidem). Moreover, this mereological reformulation brings to the fore aspects of these allegedly non-mereological notions that hitherto have been ignored. For instance, it is shown that the concept of boundary turns out to be a multiply realizable representational construct.

In contrast to the traditional account that considers spatial mereology as an abstraction of topology, the approach of this paper turns the traditional relation between mereology and topology upside down: Every Heyting mereological system $\mathrm{H}$ is shown to come along with its own canonical topological space $(\operatorname{pt}(\mathrm{H}), \mathrm{Opt}(\mathrm{H}))$ and a structure-preserving representation $\mathrm{H}--$ --pt---->Opt(H). This allows the definition of a variety of spatial concepts for the mereologically defined structure H. Thereby spatial concepts are not taken as primitives, rather, they are derived from genuine mereological concepts that are conceived of as more basic ones. In particular, topological points are not taken as primitive concepts, but show up as derived or constructed entities. Constructions like these show that the expressive power of mereology for spatial reasoning is much greater than usually is believed.

The outline of the paper is as follows. In the Section 2 we recall the basic concepts and results of set-theoretical topology and Heyting algebras to be used in the following. For more detailed presentations of the theory of Heyting algebras see (Bourceux 1994), Johnstone (1982), or Gierz et al. (2003). Section 3 seeks to show that Heyting mereological systems provide a convenient framework for dealing with at least some topological or geometrical concepts such as interior parthood and exterior contact that up to now many have considered as being beyond the conceptual reach of mereology. In Section 4 we consider co-Heyting algebras that are dual to Heyting algebras. As has been known since some time co-Heyting structures provide a natural framework for dealing with the concept of boundary. This fact can be used to build a theory of other topological concepts which in a precise sense is dual to the corresponding theory of these concepts based on Heyting systems. In Section 5 both accounts are combined to yield a representational account in which boundaries and related spatial concepts are reconstructed as representational constructs. In Section 6 the representational approach is used to show that every part $\mathrm{K} \leq \mathrm{H}$ of a Heyting algebra $\mathrm{H}$ gives rise to its own relativized concept of boundary $b_{\mathrm{K}}$ defined for the elements of $\mathrm{H}$. This may be taken as 
ultimate evidence that the concept of boundary is indeed a basically mereological concept. In Section 7 a sketch is given of how a more systematic study of spatial relations in the framework of (co-)Heyting algebras may be carried out with the help of the calculus of relations.

2. Topological Spaces and Heyting Mereology. In this section we recall the rudiments of point-set topology and the basic concepts of the theory of Heyting algebras and that will be used in the following.

A topological space $(X, O X)$ is a set $X$ provided with a family $O X$ of subsets of $X$, called open sets. OX is assumed to contain the empty set $\varnothing$ and $X$, and to be closed with respect to arbitrary unions and finite intersections. The system $(\mathrm{OX}, \subseteq$ ) is well known to be a complete Heyting algebra (see (2.2)). The set-theoretical complement of a subset a of $\mathrm{X}$ is denoted by Ca. A subset a $\subseteq \mathrm{X}$ is called closed if it is the set-theoretical complement $\mathbf{C b}$ of an open set b $\in$ OX. Obviously, a topological structure on a set X can be defined equally well by the class OX of open sets or the class CX of closed sets. The family CX of closed sets is a co-Heyting algebra (see (3.11)).

In every topological space $\mathrm{X}$ one can define the following operations on the power set PX of subsets of $X$ :

$$
\mathrm{cl}(\mathrm{a}):=\cap\{\mathrm{b} ; \mathrm{a} \subseteq \mathrm{b} \in \mathrm{CX})\} \quad \operatorname{int}(\mathrm{a}):=\cup\{\mathrm{b} ; \mathrm{b} \subseteq \mathrm{a} \text { and } \mathrm{b} \in \mathrm{OX}\}
$$

cl(a) is called the closure of $a$, and int(a) is called the interior kernel of a. The operations cl and int are interdefinable: $\operatorname{cl}(a)=\mathbf{C}(\operatorname{int}(\mathbf{C a}))$ and $\operatorname{int}(a)=\mathbf{C}(\operatorname{cl}(\mathbf{C a}))$. The (topological) boundary bd(a) of a is defined as

$$
\operatorname{bd}(\mathrm{a}):=\operatorname{cl}(\mathrm{a}) \cap \operatorname{cl}(\mathbf{C a})
$$

A subset $a \in O X$ of a topological space $(X, O X)$ is called regular open if $a=\operatorname{int}(c 1(a))$. The set of regular open sets is denoted by $\mathrm{O}^{*} \mathrm{X}$ and well known to be a complete Boolean lattice. Dually, $a$ set $b \in C X$ is regular closed if $b=\operatorname{cl}(\operatorname{int}(b))$. The set of regular closed sets is denoted by $\mathrm{C}^{*} \mathrm{X}$ and known to be a complete Boolean lattice. $\mathrm{O} * \mathrm{X}$ and $\mathrm{C}^{*} \mathrm{X}$ are canonically isomorphic by the maps $\mathrm{O}^{*} \mathrm{X}----\mathrm{cl}----->\mathrm{C} * \mathrm{X}$ and $\mathrm{C}^{*} \mathrm{X}-----i n t----->\mathrm{O} * \mathrm{X}$.

A useful introduction to the general background of lattice theory and topology to be presupposed in the following is the book of Davey and Priestley (1990). More advanced treatments 
of Heyting algebras, topology, and category theory are offered by Borceux (1994), Johnstone (1982), and Mac Lane and Moerdijk (1992).

After these preparations we are ready to present the definition of the key concept of this paper:

(2.2) Definition. Let $(\mathrm{H}, \leq)$ be a complete lattice. The supremum of a set $\mathrm{K} \subseteq \mathrm{H}$ is denoted by SUPK. For $\mathrm{a} \in \mathrm{H}$ the set $\{\mathrm{a} \wedge \mathrm{k} ; \mathrm{k} \in \mathrm{K}\}$ is denoted by a $\wedge \mathrm{K}$. The lattice $\mathrm{H}$ is a (complete) Heyting algebra if and only if for all $\mathrm{a} \in \mathrm{H}$ and all $\mathrm{K} \subseteq \mathrm{H}$ the following law holds:

$$
\mathrm{a} \wedge \mathrm{SUPK}=\mathrm{SUP}(\mathrm{a} \wedge \mathrm{K})
$$

$\operatorname{SUP}\{x ; x \wedge a \leq b\}$ is denoted by $a \Rightarrow b$ and called the Heyting complement of a relative $b$. The element $\mathrm{a} \Rightarrow 0$ is denoted by $\mathrm{a}^{*}$ and called the Heyting complement of $\mathrm{a}$. It is the largest element of $\mathrm{H}$ disjoint from a.

(2.3) Examples of Heyting algebras.

(1) The linear orders $(\mathrm{n}, \leq):=\{0,1, \ldots, \mathrm{n}-1, \leq\}, \mathrm{n} \in \mathbf{N}$, with the order $0<1<\ldots<\mathrm{n}-1$ are Heyting algebras.

(2) More generally, every finite distributive lattice $(\mathrm{D}, \leq)$ is a Heyting algebra.

(3) If $(\mathrm{X}, \mathrm{OX})$ is a topological space the lattice $(\mathrm{OX}, \subseteq)$ of open sets is a Heyting algebra.

(4) The power set $(\mathrm{PX}, \subseteq)$ of a set $\mathrm{X}$ is a Heyting algebra.

(5) If $\mathrm{G}$ is a simple, undirected graph, then the set $\mathrm{SUB}(\mathrm{G})$ of subgraphs of $\mathrm{G}$ is a Heyting algebra.

(2.4) Proposition (Borceux 1994, chapter 1, 1.2.3. - 1.2.8). For all elements a, b of a Heyting algebra $\mathrm{H}$ the following relations hold:

(1) $\quad a \leq b$ iff $(a \Rightarrow b)=1$.

(2) $\quad \mathrm{a} \Rightarrow(\mathrm{b} \wedge \mathrm{c})=(\mathrm{a} \Rightarrow \mathrm{b}) \wedge(\mathrm{a} \Rightarrow \mathrm{c})$.

(3) $\quad \mathrm{a} \wedge \mathrm{a}^{*}=0$.

(4) $\quad \mathrm{a} \leq \mathrm{a}^{* *}$.

(5) $\quad \mathrm{a}^{* * *}=\mathrm{a}^{*}$.

(6) $\quad(a \vee b)^{*}=a^{*} \wedge b^{*}$. (De Morgan law)

(7) $\quad(a \wedge b)^{*} \geq a^{*} \vee b^{*}$

(8) $\quad(\mathrm{a} \wedge \mathrm{b})^{* *}=\mathrm{a}^{* *} \wedge \mathrm{b}^{* *}$.

(9) $\quad a^{* *} \vee b^{* *} \leq(a \vee b)^{* *}$. 
(2.5) Proposition (cf. Borceux 1994, chapter 1, 1.2.11). A Boolean algebra (B, $\leq$ ) is a Heyting algebra which satisfies the following two equivalent conditions:

(1) $\quad \mathrm{a} \vee \mathrm{a}^{*}=1$, for all $\mathrm{a} \in \mathrm{B}$.

(2) $\quad \mathrm{a}=\mathrm{a}^{* *}$, for all $\mathrm{a} \in \mathrm{B}$.

\section{(2.6) Examples of Boolean Algebras.}

(1) $(2, \leq)$ is a Boolean algebra. For $\mathrm{n} \geq 3(\mathrm{n}, \leq)$ is not a Boolean algebra.

(2) If $(\mathrm{X}, \mathrm{OX})$ is a topological space, the algebra $\mathrm{O} * \mathrm{X}$ of regular open sets is a Boolean algebra. Analogously, the algebra $C^{*} \mathrm{X}$ of regular closed sets is a Boolean algebra. The algebra CX of closed sets is usually not a Boolean algebra.

(3) If $\mathrm{X}$ is a set, then the power set $(\mathrm{PX}, \subseteq)$ is a Boolean algebra.

(4) The Lindenbaum algebras in classical logic are Boolean algebras.

After these preparatory definitions we are ready to define the first topological concept that allegedly is outside the conceptual reach of mereology, to wit, the concept of connectedness:

(2.7) Definition (Connectedness, Wholeness). An element a of a lattice $(\mathrm{L}, \leq)$ is connected if and only if there do not exist disjoint elements $b, c \neq 0$ such that $b \vee c=a$. Otherwise, $a$ is called disconnected or scattered. A connected element a is also called a whole. The lattice $\mathrm{L}$ is called connected if and only if its top element $1_{\mathrm{L}}$ is connected.

Whether this definition is a ,good“ definition of the spatial notion of „connectedness“, has to be checked against examples that we intuitively judge as clear-cut cases of being connected or being disconnected, respectively. With respect to the standard examples of connected open sets of Euclidean space (E, OE) definition (2.7) scores well, since exactly those regions of a topological space that are intuitively considered as connected come out as connected according to (2.7). This should come out as no surprise: the definition of connectedness via the Heyting algebra OE just is the standard definition of topological connectedness. The important point of this elementary observation is that the definition (2.7) makes sense for any lattice regardless whether its elements are point sets (as is the case for OX) or not, since it only uses lattice-theoretical concepts of join (union) and meet (intersection). In particular this means that the concept of connectedness is definable for every Heyting algebra. 
The neat relationship between between mereology and topology with respect to the notion of connectedness depends on the non-Boolean character of the Heyting algebras involved. If we had opted for a Boolean framework (which is, after all, a special case of the Heyting one) the definition of connectedness and other spatial concepts becomes trivial and we would have had to introduce some extra spatial concept such as non-tangential parthood or contact, to deal with spatial problems.

Indeed, according to (2.7) Boolean algebras come out as quite disconnected: Take, for instance, the Boolean algebra $(\mathrm{PX}, \subseteq$ ) of the power set PX of a set $\mathrm{X}$. Then the only connected elements of the Boolean lattice PX are the singletons $\{\mathrm{x}\}$ of the element $\mathrm{x}$ of $\mathrm{X}$. In lattice theoretical terms, the singletons $\{x\}$ are the atoms of PX. More generally, the only connected parts of Boolean algebras are its atoms. As is well-known, there are even Boolean algebras that have no atoms at all and may therefore be characterized as totally disconnected. ${ }^{2}$ On the other hand, even the rather special Heyting algebras $(\mathbf{n}, \leq)$ have connected elements that are not atoms $(n \geq 3)$. Summarizing we may say that Booleaness and connectedness are opposed notions.

The definition (2.7) captures well the meaning of connectedness not only for ordinay space (and other topological spaces), its domain of applications goes far beyond that. For instance, take simple undirected graphs. In an intuitive sense a graph G may be considered as net whose parts are its subgraphs. As is easily shown this idea can be rendered precise making $G$ a Heyting mereological system with $\mathrm{G}$ as top element and the empty graph as bottom element. We we have strong intuitions which subgraphs of $G$ are connected and which are not. Also in this case, (2.7) gives the intuitively correct results. These examples may suffice to render plausible the thesis that (2.7) yields a good, purely mereological definition of the concept of connectedness.

Before we go on in the task of unfolding the variety of spatial concepts that can be formulated in the framework of Heyting mereology it seems appropriate to address a strong thesis on the conceptual limitations of mereology that has been put forward time and again by authors such as Casati, Varzi, Smith and others. According to these authors it is misleading to subscribe to the „rather widespread tendency to present mereology as a theory of parts and wholes, [since] the latter notion cannot be defined in terms of the former." (Casati and Varzi 1999, 11). Even worse, mereology is said not to be able to distinguish between „good“ and „bad wholes“, the latter consisting of ,scattered or ill-asserted entities ... by thinking exclusively in terms of parthood." (ibid.) 
As the main culprit for this deficiency Casati and Varzi identify the inability of mereology to come to terms with the concept of connectedness. Of course, they point out, connectedness may be defined in topological terms (or possibly otherwise) but all attempts to define it in purely mereological terms are doomed to fail, or, in any case, have failed up to now. As a prominent example they present Whitehead's account of connectedness in some detail, but also all other different mereological systems that are discussed in their book, don't score better. Definition (2.7) refutes their theses: As shown above, Heyting mereology successfully deals with the problem of defining connectedness, simply because the mereological definition (2.7) of connectedness is identical with the allegedly topological definition of this concept. The reason is that for (reasonably well-behaved) topological spaces their spatial structure is encapsulated in the mereological (lattice-theoretical) structure of their Heyting algebras of open sets (Gierz et al. 2003, Johnstone 1982, Mac Lane and Moerdijk 1992). In other words, under mild restrictions, a Heyting algebra $(H, \leq)$ uniquely defines a topological space $(p t(H)$, $\mathrm{O}(\mathrm{pt}(\mathrm{H}))$ such that its point set $\mathrm{pt}(\mathrm{H})$ and its topological structure can be defined in purely mereological terms. ${ }^{3}$ This applies in particular to the Euclidean spaces (E, OE) and the Heyting algebras $\mathrm{OE}$. Hence one may conjecture that non-Boolean Heyting algebras $(\mathrm{H}, \leq)$ provide a convenient framework for dealing with topological concepts. The following sections seek to corroborate this by the reconstruction of further topological concepts in the framework of a (non-Boolean) Heyting algebras.

3. Spatial Concepts in Heyting Algebras. In this section the most important spatial concepts are shown to be definable in the framework of Heyting algebras (Actually, much can be formulated even in the wider framework of distributive lattices but we will not dwell upon this generalization in detail.

(3.1) Definition. Let $(\mathrm{D}, \leq)$ be a distributive lattice, and $\mathrm{a}, \mathrm{b} \in \mathrm{D}$. Then a is an interior (or non-tangential) part of $\mathrm{c}$, to be denoted by $\mathrm{a}<<\mathrm{c}$ if and only if

$$
\mathrm{a}<<\mathrm{c}:=\mathrm{a} \leq \mathrm{c} \text { and } \exists \mathrm{b} \text { such that } \mathrm{a} \wedge \mathrm{b}=0 \text { and } \mathrm{b} \vee \mathrm{c}=1 \text {. }
$$

If $\mathrm{a} \leq \mathrm{c}$ and $\mathrm{a}$ is not an interior part of $\mathrm{c}, \mathrm{a}$ is called a tangential part of $\mathrm{c} .^{4}$ 
Applying (3.1) to the Heyting algebra OE of open sets of Euclidean space E yields the results that one intuitively expects. In the following we will consider the interior parthood relation $<<$ only for Heyting algebras. Definition (3.1) shows why Boolean algebras B cannot distinguish between tangential and non-tangential parts: Assume $\mathrm{a}, \mathrm{b} \in \mathrm{B}$ and $\mathrm{a} \leq \mathrm{b}$. Since B is Boolean, a has a complement $a^{*}$ with $a \wedge a^{*}=0$ and $a^{*} \vee a=1$. In other words, $a \leq b$ entails that $a^{*} \vee b$ $=1$. Hence the relations $<<$ and $\leq$ coincide for Boolean algebras.

(3.2) Proposition (cf. Johnstone 1982, III, 1. 81f.). If (D, $\leq$ ) is a distributive lattice the interior parthood relation $<<$ has the following properties:

(1) $\mathrm{a}<<\mathrm{a}$ if and only if a has a complement.

(2) $\mathrm{a}<<\mathrm{b}$ implies $\mathrm{a} \leq \mathrm{b}$.

(3) $\quad$ a $\leq$ b $<<$ c $\leq$ d implies a $<<$ d.

The interior parthood relation $<<$ defined by (3.1) behaves well with respect to finite suprema: (3.3) Proposition. The interior parthood relation (3.1) of a distributive lattice D is multiplicative in the sense that $\mathrm{a}_{1}, \mathrm{a}_{2}<<\mathrm{c}$ entails $\mathrm{a}_{1} \mathrm{v} \mathrm{a}_{2}<<\mathrm{c}$.

Proof. Assume $a_{1} \wedge b_{1}=a_{2} \wedge b_{2}=0$ and $b_{1} \vee c=b_{2} \vee c=1$. By (2.4)(6) for $b_{1} \wedge b_{2}$ one obtains $\left(a_{1} \vee a_{2}\right) \wedge b_{1} \wedge b_{2}=0$ and $c \vee\left(b_{1} \wedge b_{2}\right)=1$, since $D$ is distributive. Hence $a_{1} \vee a_{2}<<$.

Many interior parthhood relations are multiplicative in the sense of (3.3), but it is not reasonable to expect that $<<$ respects infinite suprema, i.e. from $\mathrm{a}_{\mathrm{i}}<<\mathrm{c}, \mathrm{i} \in \mathrm{I}$ it hardly ever follows that SUP $\left\{\mathrm{a}_{\mathrm{i}} ; \mathrm{i} \in \mathrm{I}\right\}<<$ for arbitrary sets $\left\{\mathrm{a}_{\mathrm{i}} ; \mathrm{i} \in \mathrm{I}\right\}$. Counter-examples can be found already for the Heyting algebra OE of Euclidean space.

The following lemma gives a useful topological interpretation of $<<$ for Heyting algebras OX:

(3.4) Lemma. Let $(X, O X)$ be a topological space, and $a, b \in O X$. Then a $<<$ b holds if and only if $\operatorname{cl}(\mathrm{a}) \subseteq \mathrm{b}$.

Proof. Assume $\mathrm{a}<<\mathrm{b}$. By definition $\mathrm{a}^{*}=\operatorname{int}(\mathbf{C a})$, hence $\mathrm{b} \cup \operatorname{int}(\mathbf{C a})=\mathrm{X}$. Recalling that $\mathrm{cl}=$ $\mathbf{C}$ int $\mathbf{C}$ one obtains

$$
\begin{aligned}
& \mathbf{C}(\mathrm{b} \cup \operatorname{int}(\mathbf{C a}))=\varnothing \\
& \Leftrightarrow \mathbf{C b} \cap \mathbf{C}(\operatorname{int}(\mathbf{C a}))=\varnothing \\
& \Leftrightarrow \mathbf{C b} \cap \operatorname{cl}(\mathrm{a})=\varnothing \\
& \Leftrightarrow \operatorname{cl}(\mathrm{a}) \subseteq \mathrm{b} .
\end{aligned}
$$


Since for $\mathrm{a} \in \mathrm{OX} \operatorname{cl}(\mathrm{a})=\mathrm{a} \cup \mathrm{bd}(\mathrm{a})$ (3.4) gives a nice relation between the concepts of interior parthood relation $<<$ and boundary bd that will we further exploited later.

Recall that a topological space $(\mathrm{X}, \mathrm{OX})$ is regular if and only if for every closed set $\mathrm{A}$ and every point $\alpha \notin A$ there are disjoint open neighborhoods $U(A)$ of $A$ and $U(\alpha)$ of $\alpha$. The following proposition shows that the topological concept of regularity can be expressed neatly in mereological terms:

(3.5) Proposition. Let $(H, \leq)$ a Heyting algebra. $H$ is called regular if and only if every a $\in \mathrm{H}$ can be approximated by its interior parts, i.e., $a=\operatorname{SUP}\{b ; b<<a\}$. The topological space $(X$, $\mathrm{OX})$ is regular in the sense of topology if and only if OX is regular.

Proof. Let $X$ be regular (in the sense of topology) and $\alpha \in \mathrm{a} \in \mathrm{OX}$. By regularity of $\mathrm{X}$ there are disjoint open neighborhoods $\mathrm{U}(\alpha)$ and $\mathrm{V}(\mathbf{C a})$. By definition of closedness this means that $\alpha \notin \operatorname{cl}(\mathrm{V}(\mathbf{C a}))$. Again by regularity there are disjoint open neighborhood $\mathrm{W}(\alpha)$ and $\mathrm{W}(\operatorname{cl}(\mathrm{V}(\mathbf{C a}))$. This is equivalent to $\mathrm{W}(\alpha) \subseteq \mathbf{C W}(\operatorname{cl}(\mathrm{V}(\mathbf{C a}))$. This entails that $\operatorname{cl}(\mathrm{W}(\alpha)) \subseteq$ $\mathrm{CW}(\mathrm{cl}(\mathrm{V}(\mathbf{C a}))$ since $\mathbf{C W}(\mathrm{cl}(\mathrm{V}(\mathbf{C a}))$ is closed. But clearly $\mathbf{C W}(\mathrm{cl}(\mathrm{V}(\mathbf{C a})) \subseteq$ a. Hence every point $\alpha \in$ a has a neighborhood $\mathrm{W}(\alpha)$ with $\operatorname{cl}(\mathrm{W}(\alpha)) \subseteq$ a. By (3.4) this is equivalent to $\mathrm{W}(\alpha)$ $<<$ a. Hence OX is regular in the mereological sense.

On the other hand, assume OX is regular. Suppose A is a closed subset of $\mathrm{X}$ and $\alpha \notin \mathrm{A}$. Then $\alpha \in \mathrm{Ca} \in \mathrm{OX}$. By regularity in the mereological sense there is an open neighborhood $\mathrm{V}(\alpha)$ with $\operatorname{cl}(\mathrm{V}(\alpha)) \subseteq \mathbf{C A}$. This is equivalent to $\mathrm{A} \subseteq \operatorname{Ccl}(\mathrm{V}(\alpha))$. Since clearly $\alpha \notin \operatorname{cl}(\mathbf{C c l}(\mathrm{V}(\alpha)))$ there is an open neighborhood $\mathrm{W}(\alpha) \subseteq \operatorname{Ccl}(\operatorname{Ccl}(\mathrm{V}(\alpha)))$. But then $\mathrm{W}(\alpha)$ and $\operatorname{Ccl}(\mathrm{V}(\alpha))$ are disjoint open neighborhoods of $\alpha$ and $\mathrm{A}$. Hence $(\mathrm{X}, \mathrm{OX})$ is regular in the familiar topological sense.

For regular Heyting algebras $(\mathrm{H}, \leq)$ the familiar parthood relation $\leq$ may be defined in terms of the interior parthood relation $<<$ :

(3.6) Proposition. Let $(\mathrm{H}, \leq)$ be regular Heyting algebra. Then the following holds:

$$
\mathrm{a} \leq \mathrm{b} \Leftrightarrow(\mathrm{x})(\mathrm{x}<<\mathrm{a} \Rightarrow \mathrm{x}<<\mathrm{b})
$$

Proof: Assume $\mathrm{a} \leq \mathrm{b}$ and $\mathrm{x}<<\mathrm{a}$. Then by (3.2)(3) $\mathrm{x}<<\mathrm{b}$. On other hand assume $\{\mathrm{x} ; \mathrm{x}<<\mathrm{a}\}$ $\subseteq\{\mathrm{x} ; \mathrm{x}<<\mathrm{b}\}$. Since $\mathrm{H}$ is regular this entails $\mathrm{a}=\operatorname{SUP}\{\mathrm{x} ; \mathrm{x}<<\mathrm{a}\} \leq \operatorname{SUP}\{\mathrm{x} ; \mathrm{x}<<\mathrm{b}\}=\mathrm{b}$. 
(3.7) Definition. Let $(H, \leq)$ be a Heyting algebra. The elements $a, b \in H$ are separated from each other if and only if they are disjoint and moreover satisfy the requirement:

$$
\exists \mathrm{c}, \mathrm{d}(\mathrm{c} \wedge \mathrm{d}=0 \text { and } \mathrm{a}<<\mathrm{c} \text { and } \mathrm{b}<<\mathrm{d})
$$

Evidently, for Boolean algebras, disjointness and being separated coincide. For $\mathrm{H}=\mathrm{OX}$ we obtain by (3.4) that $a$ and $b$ are separated if and only if $\operatorname{cl}(a) \cap \operatorname{cl}(b) \neq \varnothing$.

Another important topological concept that can be reformulated in the algebraic framework of Heyting algebras is compactness:

(3.8) Definition. A part $\mathrm{a} \in \mathrm{H}$ is compact if and only if for all $\mathrm{K} \subseteq \mathrm{H}$ with a $<<\mathrm{SUPK}$ there is a finite subset $\left\{b_{1}, \ldots, b_{n}\right\} \subseteq \mathrm{K}$ with $a<<b_{1} \vee \ldots \vee b_{n}$. The Heyting system $(H, \leq)$ is said to be compact if and only if $1_{\mathrm{H}}$ is compact.

(3.9) Example. (i) The Euclidean plane E is not compact with respect to the standard Euclidean topology OE. (ii) The sphere $S^{2}:=\left\{(x, y) ; x^{2}+y^{2}=1\right.$ is compact in the standard topology of $\mathrm{OS}^{2}$ induced by the inclusion $\mathrm{S}^{2} \subseteq \mathbf{R}^{3}$.

Compact parts may be considered as „small“ in the sense of being bounded. For Euclidean spaces (E, OE) a part a is compact in the sense of (3.8) if and only if ist closure cl(a) is bounded in the familiar sense that it is contained in some disk $D(r)$ of finite radius $r$. As it should be, half-planes and other intuitively unbounded subsets come out as large, i.e. they are not compact in the sense of (3.8).

Let us finally mention another useful descendent of interior parthood, to wit, the contact relation $\mathrm{C}$ which has often been claimed to be unreachable for mereology:

(3.10) Definition. The parts $\mathrm{a}, \mathrm{b} \in \mathrm{H}$ are in contact, denoted as $\mathrm{aCb}$, if and only if

$$
\mathrm{a}, \mathrm{b} \neq 0 \text { and not }\left(\mathrm{a}<<\mathrm{b}^{*}\right) \text {. }
$$

The following remarks on (3.10) may be in order. First note that (3.10) is symmetric: by definition $\mathrm{a}<<\mathrm{b}^{*}$ obtains if and only if $\mathrm{a} \leq \mathrm{b}^{*}$ and $\mathrm{a}^{*} \vee \mathrm{b}^{*}=1$. This entails that $\mathrm{b} \wedge\left(\mathrm{a}^{*} \vee \mathrm{b}^{*}\right)=$ $\left.b \wedge a^{*}\right) \vee\left(b \wedge b^{*}\right)=b \wedge a^{*}$, and therefore $b \leq a^{*}$. Secondly, (3.10) is reflexive, i.e. a $C$ a. 
Assume the contrary. Then NOT(a $\mathrm{C}$ a) is equivalent to $\mathrm{a}<<\mathrm{a}^{*}$ which entails $\mathrm{a}^{*}=1$ and therefore $\mathrm{a}^{* *}=0$ and therefore $\mathrm{a}=0$. This is a contradiction.

Next consider the topological case, i.e. $a, b \in O X$ for some topological space (X, OX). According to (3.4) and the definition of the Heyting complement a* in OX the relation NOT(a $<<$ b) entails $\mathrm{a}^{*} \vee \mathrm{b} \neq 1$, i.e. $\operatorname{NOT}(\operatorname{cl}(\mathrm{a}) \subseteq \mathbf{C c l}(\mathrm{b}))$. This is equivalent to the requirement $\mathrm{cl}(\mathrm{a})$ $\cap \operatorname{cl}(b) \neq \varnothing$. But this is exactly the definition of the contact relation $\mathrm{C}$ used in RCC and similar accounts (cf. Düntsch et. al. 1999, Düntsch et al. 2001). Moreover, it is easily shown that (3.10) satisfies the standard requirements for a contact relation to be found in the literature:

(3.11) Definition. Let $(\mathrm{L}, \leq)$ be a lattice. A binary relation $\mathrm{C}$ on $\mathrm{L}$ is called a contact relation if and only if for all $\mathrm{a}, \mathrm{b}, \mathrm{c} \in \mathrm{L}$ the following requirements are satisfied:

$$
\operatorname{NOT}(0 \mathrm{C} \text { a) }
$$

(2) IF $\mathrm{a} \neq 0$ THEN a $\mathrm{C}$ a.

(3) IF a C b and b $\leq$ c THEN a C c.

(4) IF a C $(\mathrm{b} \vee \mathrm{c})$ THEN $((\mathrm{a} \mathrm{C} b)$ OR $(\mathrm{a} \mathrm{C} \mathrm{c}))$.

As is easily shown the relation defined in (3.10) is a contact relation. The point of this contact relation is that it can be defined in the framework of Heyting algebras in purely mereological terms, while in Boolean accounts the contact relation $\mathrm{C}$ is introduced as a primitive term.

As a contrast, let us now consider the Boolean case which is closely related to the trivial topological structure:

(3.12) Example. Let (X, PX) be the trivial discrete topological structure on $\mathrm{X}$. Then the closure operator $\mathrm{cl}$ is the identity. Hence by (3.4) $\mathrm{a}<<\mathrm{b} \Leftrightarrow \mathrm{a} \leq \mathrm{b}$, and the contact relation $\mathrm{C}$ of (3.10) boils down to non-trivial intersection. This is evidence that in a Boolean framework one cannot distinguish between interior parthood and ordinary parthood, at least, if no further primitive is introduced.

These propositions may suffice to render plausible the claim that there is a natural and farreaching correspondence between (non-Boolean) Heyting mereology and topology. Nevertheless, for discussing the concept of boundary it will be useful to consider not only Heyting algebras, but also co-Heyting (or Brouwer) algebras which in a precise sense are the 
duals of Heyting algebras. Informally spoken, co-Heyting algebras arise if every lattice theoretical concept in the definition of Heyting algebras is replaced by its dual. More generally, given a lattice $(\mathrm{L}, \leq)$ define the dual lattice $\left(\mathrm{L}^{\mathrm{opp}}, \leq^{\mathrm{opp}}\right)$ by the recipe that the sets of $\mathrm{L}$ and $\mathrm{L}^{\text {opp }}$ are the same, and $\leq^{\mathrm{opp}}$ is defined as $\mathrm{x} \leq^{\mathrm{opp}} \mathrm{y}:=\mathrm{x} \geq \mathrm{y}$. Clearly, $\left(\mathrm{L}^{\mathrm{oppopp}}, \leq^{\mathrm{oppopp}}\right)=$ $(\mathrm{L}, \leq)$. Then we may define:

(3.14) Definition. A co-Heyting algebra is the dual lattice $\left(\left(\mathrm{H}^{\mathrm{opp}}, \leq^{\mathrm{opp}}\right)\right.$ of a Heyting algebra $(\mathrm{H}, \leq)$.

The duality between Heyting algebras and co-Heyting algebras entails that every theorem about Heyting algebras gives rise to a dual theorem for co-Heyting algebras and vice versa (see for instance Awodey $(2010,15)$ ). Spelt out without reference to duality co-Heyting algebras may be characterized as follows:

(3.15) Definition. A co-Heyting algebra $(\mathrm{B}, \leq)$ is a complete distributive lattice $(\mathrm{B}, \leq)$ which satisfies for all $\mathrm{a} \in \mathrm{H}$ and all $\mathrm{K} \subseteq \mathrm{H}$ the following law:

$$
\mathrm{a} \vee \operatorname{INF}(\mathrm{K})=\operatorname{INF}(\mathrm{a} \vee \mathrm{K}):=\operatorname{INF}\{\mathrm{a} \vee \mathrm{k} ; \mathrm{k} \in \mathrm{K}\}
$$

For $\mathrm{a}, \mathrm{b} \in \mathrm{H}$, the element INF $\{\mathrm{x} ; \mathrm{x} \vee \mathrm{a} \geq \mathrm{b}\}$ is called the co-Heyting complement of a relative b. The element INF $\{x ; x \vee a \geq 1\}$ is denoted by $a+$ and called the co-Heyting complement of a. By definition it is the smallest element of $B$ such that $a \vee a+=1$.

It should be noted that co-Heyting complements a+ do not satisfy the conditions for Heyting algebras, and viceversa. In particular, a $\wedge$ a $+=0$ does not hold in general.

(3.16) Examples of Co-Heyting algebras.

(1) Finite distributive lattices are co-Heyting algebras.

(2) Boolean algebras are co-Heyting algebras (and Heyting algebras).

(3) The lattice $(\mathrm{CX}, \subseteq)$ of closed sets of a topological space $(\mathrm{X}, \mathrm{OX})$ is a co-Heyting algebra. Usually, it is not a Heyting algebra.

(4) The subgraphs of a graph G define a Co-Heyting algebra (and a Heyting algebra). Usually, it is not a Boolean algebra. 
At first look, treating co-Heyting algebras alongside with Heyting algebras may seem to be a unnecessary repetition and a purely formal exercise. But it is not, since the co-Heyting framework brings to the fore concepts that have remained in the dark under the Heyting perspective. As is shown in the next section, a good example for this phenomenon is the concept of boundary.

4. Spatial Concepts in Co-Heyting Algebras. Following Lawvere (1986) in this section we argue that co-Heyting algebras provide a natural framework for dealing with boundaries (cf. also Reyes and Zolfaghari (1996)). For this purpose, points are not necessary. In other words, boundaries in the co-Heyting approach are not sets of boundary points but certain special elements of co-Heyting algebras B. Nevertheless, the basic inspiration for this approach of boundaries is taken from the example of closed sets of Euclidean spaces endowed with their standard topology.

(4.1) Definition. Let $(\mathrm{B}, \leq)$ be a co-Heyting algebra. The boundary bd(a) of a $\in \mathrm{B}$ is defined as

$$
\mathrm{bd}(\mathrm{a}):=\mathrm{a} \wedge \mathrm{a}+. \diamond^{6}
$$

For the co-Heyting algebra $\mathrm{CX}$ of closed sets of a topological space (X, OX) we obtain bd(b) $=\operatorname{cl}(\mathrm{b}) \cap \operatorname{cl}(\mathbf{C b})=\mathrm{b} \cap \operatorname{cl}(\mathbf{C b})$. Typical geometrical figures such as triangles, squares, or circles of the Euclidean plane E show that (4.1) is a reasonable definition. Intuitively, boundaries have no extension, they are ,thin“. ${ }^{7}$ This is rendered precise in the framework of co-Heyting algebras by defining an element $\mathrm{a} \in \mathrm{B}$ to be thin if and only if $\mathrm{a}++=0$. Then we obtain:

(4.2) Lemma. An element a of a co-Heyting algebra B is thin if and only if it is a boundary. The set of boundaries $\operatorname{BD}(B):=\{a ; a++=0\}$ is closed with respect to finite joins $(v)$ and finite meets $(\wedge)$, and contains 0 .

Proof. Let a be thin, i.e. $\mathrm{a}++=0$. Since $1=(\mathrm{a} \vee \mathrm{a}+)$ by $(2.4)(3)^{*},(2.4)(5)^{*}$ and $(2.4)(8)^{*}$ one obtains

$$
1=1++=(a \vee a+)++=a++\vee a+++=a++v a+=0 \vee a+=a+
$$


Therefore $b d(a)=a \wedge a+=a \wedge 1=a$. In other words, if $a$ is thin it coincides with its boundary bd(a). On the other hand, for any boundary bd(a) we obtain: bd(a)++ $=(a \wedge a+)++=(a+v$ $\mathrm{a}++)+=1+=0$. Hence $\mathrm{bd}(\mathrm{a})$ is thin. The proof of the second assertion follows from $(2.4)^{*}$

(4.3) Definition. Let $(\mathrm{B}, \leq)$ be a co-Heyting algebra with boundary bd, and $\mathrm{a}, \mathrm{b}, \mathrm{c} \in \mathrm{B}$. Then spatial concepts such as connectedness, interior parthood $<_{<_{b d}}$, and exterior contact $\mathrm{EXT}_{\mathrm{bd}}$ may be defined as follows: $\mathrm{a}$ is connected $:=\mathrm{a}$ has no parts $\mathrm{b}$ and $\mathrm{c}$ such that $\mathrm{b} \vee \mathrm{c}=\mathrm{a}$ and $\mathrm{bd}(\mathrm{b}) \wedge \mathrm{bd}(\mathrm{c})=$ 0 . If a is not connected, it is called disconnected.

$a$ is an interior part of $b, a<<_{b d} b$ iff $a \leq b$ and $b d(a) \wedge b d(b)=0$.

$a$ and $b$ are in exterior contact, $a E^{2} T_{b d} b$ iff $a \wedge b \leq b d(a) \wedge b d(b) \neq 0$.

$a$ is a regular part iff $\mathrm{a}=\mathrm{a}++$.

By checking the examples of Euclidean spaces the definitions $(4.3)(1)-(4.3)(4)$ are seen to give plausible results. Hence one may conclude that co-Heyting algebras provide a reasonable framework for mereological point-free spatial reasoning.

Before we discuss in more detail the boundary operator bd, let us mention some wide-spread erroneous conceptions about the relational properties of the boundary operator to be found in the mereological literature. These mathematical errors are probably caused by not carefully distinguishing between Boolean, Heyting, and co-Heyting systems.

For instance, in many papers and books on mereology one finds the assertion that the operator bd defined for Boolean mereological systems enjoys the following properties (Varzi 1996, Casati and Varzi 1999, Smith 1996, Smith and Varzi 2000) ${ }^{8}$ :

(4.4) Alleged Properties of Boundary Operators of Boolean Mereo(topo)logical Systems. The boundary operator for Boolean mereological systems has the following properties:

(BD1) $\quad$ bd(a) $=\operatorname{bd}(\mathbf{C a})$.

$(\mathrm{BD} 2) \quad \mathrm{bd}(\mathrm{a})=\operatorname{bd}(\mathrm{bd}(\mathrm{a}))$.

(BD3) $\quad$ bd(a) $\cup b d(b)=\quad b d(a \cap b) \cup b d(a \cup b)$.

Only (BD1) is correct - almost by definition (see (2.1)), but the claims (BD2) and (BD3) are in general false. To refute (BD2) take the real line $\mathbf{R}$ endowed with the standard topology and consider the set of rational numbers $\mathbf{Q} \subseteq \mathbf{R}$. Then $\operatorname{bd}(\mathbf{Q})=\mathbf{R}$, but $\operatorname{bd}(\operatorname{bd}(\mathbf{Q}))=\operatorname{bd}(\mathbf{R})=\varnothing$. 
As a counter example for (BD3) in $\mathbf{R}$, take the real intervals $a=[0,1]$ and $b=(1,2]$. Then bd(a) $\cup b d(b)=\{0,1,2\}$ but bd(a $\cap b) \cup b d(a \cup b)=\{0,2\}$.

Smith and Varzi even claimed that the three boundary axioms (BD1) - (BD3) were equivalent to Kuratowski's closure axioms (cf. Smith and Varzi 2000, section 3.3). This is obviously wrong: The topological space $(\mathbf{R}, \mathbf{O R})$ does satisfy the four Kuratowski axioms but neither (BD2) nor (BD3). Moreover, Smith and Varzi don't even attempt to give a proof the alleged equivalence or give any hint where to find one. ${ }^{9}$

One way to correct the erroneous claims of (4.4) is to restrict the range for which they are to hold, i.e. to replace the power set PX by some smaller subset. What about the boundaries of open sets $\mathrm{a} \in \mathrm{OX}$ or closed sets $\mathrm{b} \in \mathrm{CX}$ of a topological space (X, OX)? In other words, instead of the Boolean algebra PX one may consider the Heyting algebra OX and the coHeyting algebra CX, respectively, and ask, what relations hold for the boundaries of elements $a, b,(a \cap b)$ and $(a \cup b)$ of $O X$ and $C X$, respectively. To obtain neat results one should note that for the domains OX and CX the set-theoretical complement $\mathbf{C a}$ of a has to be replaced by the Heyting complement $\mathrm{a}^{*}$ and the Co-Heyting complement a+ in order to stay inside OX and $\mathrm{CX}$, respectively. Then we obtain the following results:

(4.5) Proposition. Let a, b open (closed) subsets of a topological space (X, OX). Denote by a\# the Heyting (co-Heyting) complement $\mathrm{a}^{*}\left(\mathrm{a}^{+}\right)$of $\mathrm{a}$, respectively. Then the boundary operator bd satisfies the following conditions:

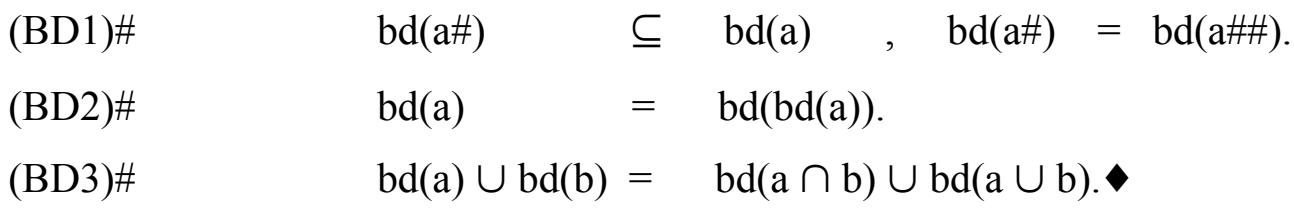

(4.6) Remark. The stronger assertions $b d(a \#)=b d(a)$ for $a=a^{*}$ and $a+$, respectively, do not hold in general. A counter-example for the Heyting algebra OE of the Euclidean plane $\mathrm{E}$ is a punctured open disk. A counter-example for the co-Heyting algebra $\mathrm{CE}$ is the closed disk to which some closed 0 -dimensional spikes or points are added.

After these clarifications concerning the boundary concept for topological systems let us engage in the task of generalizing our account of the concept of boundary from topologybased algebras $\mathrm{OX}$ and $\mathrm{CX}$ to general Heyting and co-Heyting algebras. 
The case of co-Heyting algebras is, at least at first view, simpler than that of Heyting algebras. As Lawvere already observed more than twenty years ago, a well-behaved concept of a boundary can be defined for every co-Heyting algebra $(\mathrm{B}, \leq)$, whether it has a topological origin as $\mathrm{CX}$ for some topological space $\mathrm{X}$, or not.

(4.7) Proposition. Let $(\mathrm{B}, \leq)$ be a co-Heyting algebra. Then the boundary operator bd defined by (4.1) satisfies (BD1\#) - (BD3\#).

Proof. The proofs of $(\mathrm{BD} 1 \#)$ and $(\mathrm{BD} 2 \#)$ are trivial, but that of $(\mathrm{BD} 3 \#)$ is a bit lengthy and uses the duals of the technical results for Heyting algebras in section 2. It is therefore deferred to the appendix.

Let mention now an important relation that holds for boundaries of co-Heyting algebras $\mathrm{B}$, to wit, the so called Leibniz law (cf. Lawvere (1986)):

(4.8) Proposition (Leibniz's Law). For all a, $\mathrm{b} \in \mathrm{B}$ the boundary operator bd of co-Heyting algebra B satisfies the equation:

$$
b d(a \wedge b)=(b d(a) \wedge b) \vee(b d(b) \wedge a)
$$

Proof: Using the dual de Morgan formula $(2.4)(6)^{*}(a \wedge b)+=a+\vee b+$ and the definition of the boundary operator bd one obtains

$$
\begin{aligned}
\operatorname{bd}(a \wedge b) & =(a \wedge b) \wedge(a \wedge b)+ \\
& =(a \wedge b) \wedge(a+\vee b+) \\
& =(a \wedge a+\wedge b) \vee(b \wedge b+\wedge a) \\
& =(b d(a) \wedge b) \vee(b d(b) \wedge a)
\end{aligned}
$$

The Leibniz law does not have a direct analogue in Heyting algebras, since it depends on the fact that elements and their boundaries belong to the same domain.

After having treated extensively various boundary formulas for co-Heyting algebras, it may be appropriate to eventually mention the general formula of how the topological boundaries of arbitrary sets $a, b$ and their union and intersection $a \cup b$ and $a \cap b$ are related. It does not seem to be well known among mereologists - at least to my knowledge it has never been mentioned in any mereological book or paper: 
(4.9) Proposition (A.H. Stone (Kuratowski and Mostowski 1986, 32). Let (X, OX) be a topological space. Then for all subsets $\mathrm{a}, \mathrm{b}$ of $\mathrm{X}$ the following boundary formulas holds:

$$
b d(a) \cup b d(b)=b d(a \cap b) \cup b d(a \cup b) \cup(b d(a) \cap b d(b))
$$

\section{Proof. See Appendix.}

The following remarks on (4.9) may be in order. Proposition (4.9) is not a deep result. It is proved by a lengthy but elementary calculation, using only the definitions and elementary properties of the concepts of boundary and closure. Kuratowski and Mostowski attribute to the topologist A.H. Stone, but they don't give any precise reference to Stone's work. Instead, they are content to propose the proof of (4.9) as an exercise (Kuratowski and Mostowski (1976, I. 2 (a), 32)). Since I have not found any proof of (4.9) in the standard text books on topology, for the convenience of the reader a full proof of (4.9) is given in the appendix.

Stone's equation (4.9) may be considered as a generalization for the boundary formula (4.6) which only holds either for open or closed sets a and b, respectively. As is easily seen, the extra factor „bd(a) $\cap$ bd(b)“ being present in (4.9) but not in (4.6), makes no contribution in the case of $\mathrm{a}$ and $\mathrm{b}$ both being open or closed, respectively, but is relevant for general subsets $a, b$ of $X$.

Theorem (4.9) can be used to prove that the concept of boundary can be taken as a primitive concept of topology, in the sense that the more familiar primitive concepts of open sets and closed sets can be defined in terms of bd:

(4.10) Theorem. Let X be a set. If an operator PX---bd--->PX satisfies the following set of axioms the operator $\mathrm{cl}$ defined by $\mathrm{cl}(\mathrm{x}):=\mathrm{x} \cup \operatorname{bd}(\mathrm{x})$ satisfies the four Kuratowski axioms.

$$
\begin{aligned}
& \operatorname{bd}(\varnothing)=b d(X)=\varnothing . \\
& b d(b d(a)) \subseteq b d(a) . \\
& a \subseteq b \Rightarrow b d(a) \subseteq b \cup b d(b) . \\
& b d(a) \cup b d(b)=b d(a \cap b) \cup b d(a \cup b) \cup(b d(a) \cap b d(b)) .
\end{aligned}
$$

For the closure operator cl defined in this way the open sets of the resulting topological structure are those which satisfy a $\cap$ bd(a) $=\varnothing$ while the closed sets are characterized by $\mathrm{bd}(\mathrm{c}) \subseteq \mathrm{c}$.

Vice versa, if an operator cl satisfies the four Kuratowski axioms the operator bd defined by $\operatorname{bd}(\mathrm{x}):=\operatorname{cl}(\mathrm{x}) \cap \operatorname{cl}(\mathbf{C x})$ satisfies the axioms (1) $-(4)$. 
Proof. Modulo (4.9) the proof of (4.10) is a straight-forward exercise in manipulating the definitions of the operators $\mathrm{cl}$ and $\mathrm{bd}$.

Finally, it may be observed that (4.9) can be proved for Boolean algebras $(\mathrm{B}, \leq, \mathrm{c})$ endowed with an appropriate closure operator $\mathrm{c}$ satisfying the lattice-theoretical version of the Kuratowski axioms (cf. Kuratowski and Mostowski (1976, §8, p.27)). After this disgression to the more special case of Boolean algebras, let us come back again to the more general case of co-Heyting algebras.

Even if co-Heyting algebras provide a good basis for the calculation of boundaries, one may object that co-Heyting boundaries are conceptually not always adequate. Take, for instance, the problem of modeling the border relation between, say, France and Germany. Modelling the territories of Germany and France as elements of a co-Heyting algebra, suggests that they literally share a common part, namely, the border between them. This doesn't seem conceptually very plausible. Moreover, in section 3 we defined for Heyting systems a variety of allegedly topological concepts in purely mereological terms - with the exception of the boundary concept. This evidences that the co-Heyting approach is not the only possible one. At least some spatial concepts can be defined independently of the co-Heyting approach, namely by using the Heyting approach, which, admittedly, has the shortcoming that it does not allow the definition of boundaries. The representational approach to be presented in the next section aims combines the best of the Heyting and the co-Heyting approach. In a sense, it is a means to have the cake and eat it too, i.e. to have the calculatory advantages of the CoHeyting approach but without its ontological burden, namely, to consider boundaries as genuine parts of the entities whose boundaries they are.

5. Boundaries as Representational Constructs. Although one may define for Heyting systems important topological concepts such as connectedness, interior parthood, in one point we still seem to depend on topology: Only if $\mathrm{H}$ happens to be the Heyting algebra OX of open subsets of a topological space (X, OX), we can define boundaries bd(a) for elements for open sets a with the help of a set-theoretical representation OX ----i---->PX, i.e. bd(a) $=\operatorname{cl}(a) \cap \operatorname{cl}(\mathbf{C a})$ (cf. 2.1). But for general Heyting algebras $\mathrm{H}$ such a representation seems not to be available. In this respect the co-Heyting approach seems to score better, since it allows a natural pointfree definition of boundaries independently on any underlying topological structure. 
Nevertheless, despite its simplicity, the way of how boundaries are handled in the co-Heyting framework may not be fully satisfying. Consider, for instance, the case of political or administrative borders. Even if everybody agrees that, say, France and Germany have a border, while Germany and Italy do not, one may hesitate to say that the former two countries share a common part, namely their border, while the latter two do not, as is suggested by the co-Heyting approach of boundaries.

In any case, it does not seem appropriate to ascribe to a border the same ontological robustness as to the other ordinary parts of an object, but exactly this equal treatment is suggested by the co-Heyting approach, since boundaries of objects are elements of the co-Heyting algebra alongside the objects whose boundaries they are. This is true, even if we may distinguish between ,thin“ and ,thick“ parts of a Heyting algebra according to definition (4.2). Hence the Heyting account according to which boundaries belong to a domain completely separated from the domain of the objects themselves, seems ontologically more satisfying. The only conceptual difficulty that remains is from where to get something like the settheoretical or topological representation that allows us to construct such a boundary. This difficulty will be overcome in this section: For any Heyting system $\mathrm{H}$ we construct in a natural way a set-theoretical or topological representation that allows us to define boundaries for the the elements of $\mathrm{H}$. More precisely, every Heyting algebra, comes along with its own topological space $(\operatorname{pt}(\mathrm{H}), \mathrm{O}(\mathrm{pt}(\mathrm{H}))$ and a frame map H-----f---->O(pt(H)). Depending on the type of $\mathrm{H}$ this representation is more or less faithful, i.e., it may happen that $\mathrm{f}$ is an isomorphism, under less fortunate circumstances, this representation may be trivial, in that $\mathrm{pt}(\mathrm{H})=$ $\varnothing$ and therefore will tell us nothing about $\mathrm{H}$. In this case $\mathrm{H}$ is called a Heyting algebra without points (cf. Mac Lane and Moerdijk (1992), Bourceux (1994)).

The idea for the construction of the space $(\operatorname{pt}(\mathrm{H}), \mathrm{O}(\mathrm{pt}(\mathrm{H}))$ may be traced back to M. Stone who carried it out for the special case of Boolean algebras. Only later, it was generalized to other classes of lattices, for instance, Heyting algebras. Somewhat ironically, the construction is simpler for Heyting algebras than for the original class of Boolean algebras.

A natural starting point for the task of constructing the topological space $(\mathrm{pt}(\mathrm{H}), \mathrm{O}(\mathrm{pt}(\mathrm{H}))$ is to construct the elements of $\mathrm{pt}(\mathrm{H})$. We get a hint of how to solve this problem by considering once again the special case of a trivial topological space $(\mathrm{X}, \mathrm{PX})$ and paying attention to the role, which the elements of $X$ play in distinguishing the subsets of $X$. Every point $y \in X$ may be considered as a function $\mathrm{f}_{\mathrm{y}}$ : $P X----->2$ defined by $\mathrm{f}_{\mathrm{y}}(\mathrm{a})=1$ iff $\mathrm{y} \in \mathrm{a}$, and $\mathrm{f}_{\mathrm{y}}(\mathrm{a})=0$, otherwise, $a \in P X$. As is easily observed, this renders $\mathrm{f}_{\mathrm{y}}$ a Boolean homomorphism between the 
Boolean algebras PX and 2. Moreover, as can be easily checked this correspondence between „real points“ $y \in X$ and „functional points" $f_{y}$ is a $1-1$ correspondence.

It is tempting to establish an analogous correspondence between ,real points“ $\mathrm{y}$ and ,abstract functional points“ $f_{y}$ not only for Boolean algebras and Boolean maps but also for more general lattices, and appropriate lattice-theoretical homomorphisms between them. For Heyting algebras the following definition turns out to be appropriate:

(5.1) Definition. Let $\mathrm{H}$ and $\mathrm{K}$ be a Heyting algebra. A frame map H----f-----> K is a map that preserves arbitrary joints and finite meets, i.e. $\mathrm{f}(\mathrm{SUPM})=\operatorname{SUP}(\mathrm{f}(\mathrm{M}))$ and $\mathrm{f}(\mathrm{a} \wedge \mathrm{b})=\mathrm{f}(\mathrm{a}) \wedge$ $\mathrm{f}(\mathrm{b})$, for $\mathrm{M} \subseteq \mathrm{H}$, and $\mathrm{a}, \mathrm{b} \in \mathrm{H}$. The set $\mathrm{pt}(\mathrm{H})$ of ,functional points“" of $H$ is defined as the set of frame morphisms H-----f----->2.

For the Heyting algebras OX of open sets of Hausdorff topological spaces we obtain the following pleasing result:

(5.2) Example. Let OX be the Heyting algebra of open sets of a Hausdorff topological space $(\mathrm{X}, \mathrm{OX})$. Then $\mathrm{pt}(\mathrm{OX})=\mathrm{X}$.

Proof. Let $x$ be point of $X$ and $O X----f_{x}--->2$ it characteristic function. Let $x, y \in X$ be two different points. Since $X$ is Hausdorff, there are two disjoint open neighborhoods $U(x)$ and $\mathrm{V}(\mathrm{y})$ of $\mathrm{x}$ and $\mathrm{y}$, respectively. Hence $\mathrm{f}_{\mathrm{x}}(\mathrm{U}(\mathrm{x}))=1$ and $\mathrm{f}_{\mathrm{y}}(\mathrm{U}(\mathrm{x}))=0$, and analogously for $\mathrm{V}(\mathrm{y})$. Hence the mapping $\mathrm{X}----$ pt---->ptX is a monomorphism. To prove that $\mathrm{pt}$ is also surjective, let $\mathrm{f}$ be a functional point, i.e. a frame homomorphism $\mathrm{OX}-----\mathrm{f}---->2$. Clearly, $\mathrm{f}$ is uniquely determined by $\mathrm{f}^{-1}(0):=\cup\{\mathrm{a} ; \mathrm{f}(\mathrm{a})=0\} \in \mathrm{OX}$. We want to show that the set-theoretical complement $F$ of $\mathrm{f}^{-1}(0)$ is a singleton $\{\mathrm{x}\}$. Assume the contrary, i.e. there is a different point $\mathrm{y}$ such that $\{x, y\} \subseteq F$. Since $X$ is Hausdorff there are disjoint open neighborhoods $U(x), V(y)$ of $\mathrm{x}$ and $\mathrm{y}$, respectively. Define $\mathrm{F}_{\mathrm{x}}:=\mathrm{F} \cap \mathbf{C U}(\mathrm{x})$ and $\mathrm{F}_{\mathrm{y}}:=\mathrm{F} \cap \mathbf{C V}(\mathrm{y})$. For the complements $\mathbf{C F}_{\mathrm{x}}$ and $\mathbf{C F}_{\mathrm{y}}$ one has by definition $\mathrm{f}\left(\mathrm{CF}_{\mathrm{x}}\right)=\mathrm{f}\left(\mathbf{C F}_{\mathrm{y}}\right)=1$, but by construction one has $\mathrm{f}\left(\mathrm{CF}_{\mathrm{x}} \cap\right.$ $\left.\left.\mathbf{C F}_{\mathrm{y}}\right)=\mathrm{f}\left(\mathbf{C}\left(\mathrm{F}_{\mathrm{x}} \cup \mathrm{F}_{\mathrm{y}}\right)\right)=\mathrm{f}(\mathbf{C F})\right)=\mathrm{f}\left(\mathrm{f}^{-1}(0)\right)=0$. This is a contradiction. Hence $\mathrm{F}$ is a singleton $\{\mathrm{x}\}$ and therefore the functional point $\mathrm{f}$ coincides with $\mathrm{f}_{\mathrm{x}}$. This proves that the map $\mathrm{X}$------pt---$>\operatorname{pt}(\mathrm{X})$ is surjective.

To render $\mathrm{pt}(\mathrm{H})$ a topological space one has to endow it with a topology $\mathrm{Opt}(\mathrm{H})$ : 
(5.3) Proposition (Mac Lane and Moerdijk 1992, 475-476). Let $\mathrm{H}$ be a Heyting algebra. A topology on $\mathrm{pt}(\mathrm{H})$ is defined by the following recipe. For $\mathrm{a} \in \mathrm{H}$ define $\mathrm{pt}(\mathrm{a}):=\{\mathrm{p} \in \mathrm{pt}(\mathrm{H})$; $\mathrm{p}(\mathrm{a})=1\}$. Then the Heyting algebra $\operatorname{Opt}(\mathrm{H}):=\{\operatorname{pt}(\mathrm{a}) ; \mathrm{a} \in \mathrm{H}\}$ defines a topology on the set $\operatorname{pt}(\mathrm{H})$.

Proof. One has to show that $\{\operatorname{pt}(\mathrm{a}) ; \mathrm{a} \in \mathrm{H}\}$ is closed with respect to finite intersections and arbitrary unions, and contains $\varnothing$ and $\mathrm{pt}\left(1_{\mathrm{H}}\right)$. This follows from the fact that the functional points $\mathrm{p}$ are assumed to be frame maps. Thus, for arbitrary suprema and finite infima one obtains

$$
\operatorname{pt}\left(\operatorname{SUP}\left\{\mathrm{a}_{\mathrm{i}} ; \mathrm{i} \in \mathrm{I}\right\}=\cup\left\{\operatorname{pt}\left(\mathrm{a}_{\mathrm{i}}\right) ; \mathrm{i} \in \mathrm{I}\right\} \quad, \quad \operatorname{pt}(\mathrm{a} \wedge \mathrm{b})=\operatorname{pt}(\mathrm{a}) \cap \operatorname{pt}(\mathrm{b}),\right.
$$

Moreover, one clearly has $\operatorname{pt}(0)=\varnothing$ and $\operatorname{pt}(1)=\operatorname{pt}(H)$. This shows that $\{\mathrm{pt}(\mathrm{a}) ; \mathrm{a} \in \mathrm{H}\}$ defines a topology on $\mathrm{pt}(\mathrm{H})$.

This construction of a topological space $(\operatorname{pt}(\mathrm{H}), \operatorname{Opt}(\mathrm{H}))$ for every Heyting algebra $\mathrm{H}$ puts upside down the familiar relation between mereology and topology: According to the standard account, the mereological structure of systems satisfying the axioms of General Extensional Mereology (GEM, Casati and Varzi, 1999) is an epiphenomenon of their underlying topological structure, i.e., the mereological individuals of these systems are conceived of as subsets of a given point set. ${ }^{11}$

The construction of (5.3) reverses this situation: one starts with a purely mereological structure $(\mathrm{H}, \leq)$ and then a posteriori constructs from it a topological structure $(\mathrm{pt}(\mathrm{H})$, $\operatorname{Opt}(\mathrm{H}))$, in particular a point set $\mathrm{Opt}(\mathrm{H})$, such that $\mathrm{H}$ can be represented by the topological space $(\operatorname{pt}(\mathrm{H}), \operatorname{Opt}(\mathrm{H}))$, or, more precisely, by the point-set Heyting algebra $\operatorname{Opt}(\mathrm{H}))$. Points and sets of points are not something given, rather, they arise as constructs built from mereological entities that are conceived of as conceptually more primitive.

A natural question to ask is under what conditions on the Heyting algebra $\mathrm{H}$ the map $\mathrm{H}----$ pt-$-->\mathrm{O}(\mathrm{pt}(\mathrm{H}))$ is an isomorphism. Indeed, the map pt may be trivial, i.e. it may happen that $\mathrm{H}$ is such thathas no points at all. Those Heyting algebras are said „not to have enough points“. Fortunately, this question has a very neat and satisfying answer. In order to formulate it, we need the following preparatory definition:

(5.5) Definition. Let $(\mathrm{L}, \leq)$ be a complete lattice. A subset $\mathrm{D} \subseteq \mathrm{L}$ is directed if the supremum $\operatorname{SUP}\left\{\mathrm{d}_{1}, \ldots, \mathrm{d}_{\mathrm{n}}\right\}$ of every finite subset $\left\{\mathrm{d}_{1 .}, \ldots, \mathrm{d}_{\mathrm{n}}\right\} \subseteq \mathrm{D}$ belongs to $\mathrm{D}$. The lattice $\mathrm{L}$ is conti- 
nuous if and only if for every directed $\mathrm{D} \subseteq \mathrm{L}$ and $\mathrm{x} \in \mathrm{L}$ the fact that $\mathrm{x} \leq \mathrm{SUPD}$ entails that there is $\mathrm{d} \in \mathrm{D}$ with $\mathrm{x} \leq \mathrm{d}$.

Of course, every finite lattice is continuous. But not every infinite complete lattice is continuous. A paradigmatic example is the linear $\operatorname{order}(\mathbf{Q}, \leq)$ of rational numbers. Its failure to be a continuous lattice in the sense of (5.5) is just another formulation of the assertion that it is not Dedekind-complete in the usual sense. As it should be $(\mathbf{R}, \leq)$ comes out as a continuous lattice in the sense of (5.4). Now we can state the relevant theorem:

(5.6) Theorem (Gierz et al. 2003, Theorem I-3.15, p. 101). H has enough points, i.e., H-----pt----- $>\mathrm{Opt}(\mathrm{H}))$ is an isomorphism, if and only if $\mathrm{H}$ is continuous.

Continuous lattices abound. For instance, the lattices OX of locally compact topological Hausdorff spaces (X, OX) are continuous. In terms of category theory, theorems such as (5.4) set up a isomorphism between appropriate categories of topological spaces and categories of appropriate Heyting algebras. Roughly spoken, continuous Heyting algebras and well-behaved topological spaces are therefore essentially one and the same thing. This fact lies at the heart of the mereological reconstructions of spatial concepts carried out in this paper.

It is elucidating to reconsider from the perspective of functional points the problem of defining the allegedly unmereological concept of boundary. As explained above the canonical map H---pt--->pt(H) represents an mereological individual a by the set pt(a) of frame maps $\varphi$ : H------>2 with $\varphi(a)=1$. Since the sets $\operatorname{pt}(\mathrm{a})$ are open in $\operatorname{Opt}(\mathrm{H})$ and the intersection between open sets $c$ and their boundary bd(c) is empty, boundary points $\varphi$ of a must satisfy $\varphi(a)=0$. On the other hand, $\varphi$ should be as close as possible to a. This can be rendered precise by considering the set $\varphi^{-1}(1)=\{b ; \varphi(b)=1, b \in H\}$. Then a boundary point $\varphi$ of a should satisfy the requirement that for all $b \in \varphi^{-1}(1)$ the intersection $a \wedge b$ is non-empty. Indeed, this is just the familiar definition of a boundary point of a as a point all of whose neighborhoods have a non-empty intersection with the region a and its complementary region $\mathbf{C a}$. It should be noted that this definition does not use any topological concept.

Having constructed for every Heyting mereological system $(H, \leq)$ a topological space $(\mathrm{pt}(\mathrm{H})$, $\mathrm{O}(\mathrm{pt}(\mathrm{H}))$ and a representation of $\mathrm{H}$ in $\mathrm{O}(\mathrm{pt}(\mathrm{H}))$, the definition of a natural concept of a representational boundary for the elements of any Heyting algebra $\mathrm{H}$ is at hands: 
(5.7) Proposition. Let $\mathrm{H}$ be a Heyting algebra and $\mathrm{H}-----p t---->\mathrm{O}(\mathrm{pt}(\mathrm{H}))$ its topological representation. Then the boundary $b_{\mathrm{pt}}(\mathrm{a})$ of $\mathrm{a} \in \mathrm{H}$ is defined as $\mathrm{bd}(\mathrm{pt}(\mathrm{a}))$. The boundary operator $\mathrm{bd}_{\mathrm{pt}}$ satisfies the standard law:

$$
b d_{p t}(a \wedge b) \cup b d_{p t}(a \vee b)=b d_{p t}(a) \cup b d_{p t}(b)
$$

Proof: The representation pt is a lattice homomorphism. Hence we get

$$
\begin{aligned}
\mathrm{bd}_{\mathrm{pt}}(\mathrm{a} \wedge \mathrm{b}) \cup \mathrm{bd}_{\mathrm{pt}}(\mathrm{a} \vee \mathrm{b}) & =\operatorname{bd}(\operatorname{pt}(\mathrm{a} \wedge \mathrm{b})) \cup \mathrm{bd}(\operatorname{pt}(\mathrm{a} \vee \mathrm{b})) \\
& =\mathrm{bd}(\operatorname{pt}(\mathrm{a}) \wedge \operatorname{pt}(\mathrm{b})) \cup \mathrm{bd}(\operatorname{pt}(\mathrm{a}) \vee \operatorname{pt}(\mathrm{b})) \\
& =\operatorname{bd}(\operatorname{pt}(\mathrm{a})) \cup \mathrm{bd}(\operatorname{pt}(\mathrm{b})) \\
& =b d_{p t}(\mathrm{a}) \cup b d_{p t}(\mathrm{~b}) .
\end{aligned}
$$

This definition of a representationally constituted boundary is ,correct“ since for good topological spaces (X, OX) such as Euclidean spaces whose Heyting algebra OX of open sets is continuous, the representational boundary $b_{\mathrm{pt}}(\mathrm{a})$ of $\mathrm{a} \in \mathrm{OX}$ defined by the isomorphism $\mathrm{OX}$ ---pt---->O(pt $(\mathrm{OX}))$ coincides with the familiar topological boundary bd(a).

The representational account leads to an „ontology light“, so to speak, in that borders are conceptualized as representational constructs, and not as basic entities on a par with those entities whose borders they are. For instance, although Germany and France have a common border, this need not to be interpreted as the fact that they share a common part, to wit, their border. Evidently, this part would not be of the same kind as, say, we consider the Palatinate being a part of Germany and Burgundy being a part of France. Rather, the „border parts“ of countries arise as conceptual constructs resulting from certain representations. Therefore, they lack the ontological dignity of „real“ parts. They are „ontologically thin“ theoretical constructs.

Finally let us take up a loose end from section 3. There we already defined the concept of spatial relations such as interior parthood, exterior contact and others on the basis of the mereological definition of interior parthood (3.1). It is natural to ask how the spatial concepts of section 3 are related to the representationally constructed ones introduced in this section. The answer is as neat as possible: 
(5.8) Proposition. Let $(\mathrm{H}, \leq)$ be a Heyting algebra and denote by $<<$ its interior parthood relation defined by (3.1). If $\mathrm{H}-----p t---->\mathrm{O}(\mathrm{pt}(\mathrm{H}))$ is a topological representation and $<<_{\mathrm{pt}}$ it associated interior parthood relation then $\mathrm{a}<<\mathrm{b}$ entails $\mathrm{a}<<_{\mathrm{pt}} \mathrm{b}$.

Proof. Suppose $a<<b$. This is to say that there is $c \in H$ such that $a \wedge c=0$ and $c \vee b=1$. Since pt is a lattice homomorphism one obtains

$$
\operatorname{pt}(\mathrm{a} \wedge \mathrm{c})=\operatorname{pt}(\mathrm{a}) \wedge \operatorname{pt}(\mathrm{c})=\operatorname{pt}(0)=0, \text { and } \operatorname{pt}(\mathrm{c} \vee \mathrm{b})=\operatorname{pt}(\mathrm{c}) \vee \operatorname{pt}(\mathrm{b})=\operatorname{pt}(1)=1
$$

Hence pt(c) may be taken as an element that ensures that $\operatorname{pt}(\mathrm{a})<<_{\mathrm{pt}} \mathrm{pt}(\mathrm{b})$

In sum, spatial relations defined on the base of (3.1) are compatible with their representationally defined analogues defined with the help of representations H-----pt---->O(pt $(H)$. Thus the representational approach may be considered a natural continuation of the orginal one base on Heyting algebras. If $\mathrm{H}$ happens to be a topological Heyting algebra $\mathrm{OX}$ then both boundary concepts coincide.

6. Relativizing Boundaries. In this section we further elaborate the idea of conceiving boundaries as representational constructs. For every element a of a Heyting algebra $\mathrm{H}$ boundary operators $b d_{\downarrow a}$ and $b d_{a \uparrow}$ will be defined that concentrate, in a way to be specified, on the boundary relations that occur near a. Hence, replacing the ordinary boundary by its ,arelativized" versions $b d_{\downarrow}$ and $b d_{a \uparrow}$ corresponds to narrowing down the focus of attention to what happens around the parts a of $\mathrm{H}$.

First recall that every part a of a mereological system $(\mathrm{H}, \leq)$ defines its own mereological system consisting of its parts defined by

$$
\downarrow \mathrm{a}:=\{\mathrm{x} ; \mathrm{x} \in \mathrm{H} \text { and } \mathrm{x} \leq \mathrm{a}\}
$$

Clearly, if $\mathrm{H}$ is a Heyting system the $\downarrow$ a are Heyting systems as well, namely, the systems of all parts of the elements $a \in H$. The systems $\downarrow$ a are endowed with frame epimorphisms H---$f_{a^{----}}>\downarrow$ a defined by $f_{a}(x):=a \wedge x$. Then the concatenation

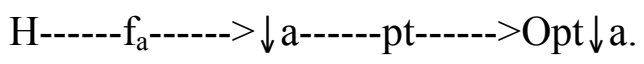

defines a boundary operator $b d_{\downarrow a}$ for the Heyting algebra $H$. The operator $b d_{\downarrow a}$ is called the $\downarrow$ a-relativized boundary of $\mathrm{H}$. 
The system $(\downarrow \mathrm{a}, \leq)$ is not the only mereological system defined by a $\in$ H. Dually, a part a defines another mereological system by

$$
\uparrow a:=\{x ; x \in H \text { and } x \geq a\}
$$

consisting of the elements of $\mathrm{H}$ that contain a as a part. Completely analogous to the systems $(\downarrow \mathrm{a}, \leq)$ the systems $\uparrow \mathrm{a}$ are endowed with frame maps $\mathrm{H}----\mathrm{g}_{\mathrm{a}}---->\uparrow \mathrm{a}$ defined by $\mathrm{g}_{\mathrm{a}}(\mathrm{x}):=\mathrm{x} \vee$ a. Then the concatenation $\mathrm{H}----\mathrm{g}_{\mathrm{a}^{-----}>\uparrow a----p t---->O p t}(\uparrow a)$ defines a boundary operator $\mathrm{bd}_{\uparrow a}$ for the Heyting algebra $\mathrm{H}$. This boundary operator is called the $\uparrow$ a-relativized boundary of $\mathrm{H}$. By its very construction these boundary operators satisfy the fundamental laws of boundaries (4.4) (BD1) - (BD3).

This relativization of boundaries with respect to the parts $\uparrow \mathrm{a}$ and $\downarrow \mathrm{a}$ of $\mathrm{H}$ can be generalized further. The basic ingredients for the definition of the operators $b d_{\downarrow a}$ and $b d_{\uparrow a}$ were the frame

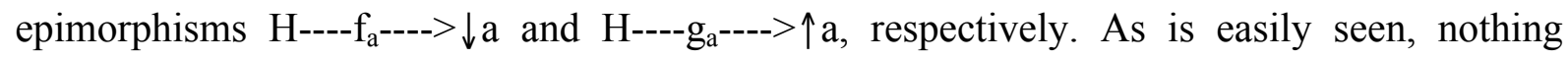
depends on the special structure of $\downarrow a$ and $\uparrow a$, the recipe works for any Heyting algebra $K$ endowed with a frame epimorphism H-----h---->K. Given such a map the K-relativized boundary operator $\mathrm{bd}_{\mathrm{K}}$ is defined with the help of the concatenation $\mathrm{H}----\mathrm{h}--->\mathrm{K}----\mathrm{pt}---$ $>$ OptK by $b_{K}(a):=\operatorname{bd}(\operatorname{pt}(h(a)))$. Indeed, one may develop a full-fledged mereology of Heyting algebras which takes as parts of $\mathrm{H}$ any Heyting algebra $\mathrm{K}$ endowed with a frame epimorphism frame H-----h---->K. As a non-trivial result one obtains that the resulting mereological system PART(H) of $\mathrm{H}$ has again the structure of a Heyting algebra. Remarkably, it turns out that the $\uparrow a$ and $\downarrow$ a are not the only parts of PART(H) (cf. Borceux 1994, Johnstone 1982, Lawvere and Rosebrugh 2003).

As a final result on the issue of relativizing boundaries we may therefore state the following:

(6.5) Proposition (K-relative Boundaries). Let $\mathrm{H}------\mathrm{g}----->\mathrm{K}$ be a frame epimorphism of $\mathrm{H}$ onto K. Then the concatenation of maps

$$
\text { H------f----->K------pt------>Opt(K) }
$$

defines a boundary operator $b d_{K}$ by $b d_{K}(a):=b d(p t(f(a)))$. Since $f$ and pt preserve finite meets and joins and the concatenation $\mathrm{pt} \cdot \mathrm{f}$ maps the elements of $\mathrm{H}$ onto open subsets, $\mathrm{bd}_{\mathrm{K}}$ satisfies the fundamental laws, in particular

$$
b d_{K}(a \vee b) \cap b d_{K}(a \wedge b)=b d_{K}(a) \cup b d_{K}(b)
$$


Summarizing one can assert that the genuinely spatial concept of boundary is deeply rooted in the mereological structure of Heyting systems $\mathrm{H}$ in that every part $\mathrm{K}$ of $\mathrm{H}$ defines in a natural way well-behaved its specific boundary operator $b_{\mathrm{K}}$ for the elements of $\mathrm{H}$. If $\mathrm{H}$ happens to be the Heyting algebra OX of open sets of some topological space (X, OX) and $\mathrm{K}=\mathrm{OX}$, then the relativized boundary operator $b_{\mathrm{OX}}$ is just the familiar topological boundary operator bd of the topological space $(\mathrm{X}, \mathrm{OX}){ }^{12}$ In other words, there is comprehensive mereological grounding of the allegedly non-mereological spatial concept of boundary.

7. Concluding Remarks. The previous sections of this paper concentrated on some spatial key concepts such as connectedness, interior parthood, contact and the like. The main aim was to show that - contrary to common wisdom - these concepts can be defined in purely mereological terms. This paper did not offer a systematic account of spatial concepts that can be defined in a mereological framework. At the end of this contribution it may be expedient, however, to give a sketch of how a more systematic account spatial mereological concepts may look like. For this purpose it is useful to get an idea of how to generate further spatial concepts from those that are already available. For this we concentrate on binary spatial concepts and tap the sources of the calculus of relations (cf. Tarski (1941), Düntsch et al. (1999), Düntsch et al. 2001).

Let $\mathrm{REL}(\mathrm{H}):=\{\mathrm{R} ; \mathrm{R} \subseteq \mathrm{H} \times \mathrm{H}\}$ denote the set of binary relations on $\mathrm{H}$. Clearly, REL(H) as being the power set $\mathrm{P}(\mathrm{H} \times \mathrm{H})$ of $\mathrm{H} \times \mathrm{H})$ is a Boolean algebra with respect to the standard settheoretical operations of union $(\cup)$, intersection $(\cap)$, and set-theoretical complement $\mathbf{C}$. But there are other operations defined on $\operatorname{REL}(\mathrm{H})$ that are not present in general Boolean algebras. First, there is the idempotent operator of conversion: for the binary relation $\mathrm{R}$ the converse relation $\mathrm{R}^{\circ}$ is defined as $\mathrm{R}^{\circ}:=\{(\mathrm{b}, \mathrm{a}) ;(\mathrm{a}, \mathrm{b}) \in \mathrm{R}\}$. Clearly $\mathrm{R}^{\circ \circ}=\mathrm{R}$.

The most interesting operation on $\operatorname{REL}(\mathrm{H})$, however, is the relational product:

(7.1) Definition. Let $\mathrm{R}$ and $\mathrm{S}$ be binary relations on $\mathrm{H}$. Then the relational product $\mathrm{R} \cdot \mathrm{S}$ is defined as

$$
\mathrm{R} \cdot \mathrm{S}:=\{(\mathrm{x}, \mathrm{z}) ; \exists \mathrm{y}((\mathrm{x}, \mathrm{y}) \in \mathrm{R} \operatorname{AND}(\mathrm{y}, \mathrm{z}) \in \mathrm{S})\}
$$

Clearly, the concatenation of relational product and conversion satisfy $(\mathrm{R} \bullet \mathrm{S})^{\circ}=\mathrm{S}^{\circ} \bullet \mathrm{R}^{\circ}$ 
(7.2) Definition (Examples of binary spatial relations). For a Heyting algebra $(H, \leq)$ the $\mathrm{REL}(\mathrm{H})$ contains (among many others) the following relations:

$$
\begin{array}{ll}
\mathrm{P}:=\{(\mathrm{a}, \mathrm{b}) ; \mathrm{a} \leq \mathrm{b}\} & (\text { Part) } \\
\mathrm{PP}:=\{(\mathrm{a}, \mathrm{b}) ; \mathrm{a}<\mathrm{b}\} & \text { (Proper Part) } \\
\mathrm{ID}:=\{(\mathrm{a}, \mathrm{a}) ; \mathrm{a} \in \mathrm{H}\} & \text { (Identity) } \\
\mathrm{O}:=\{(\mathrm{a}, \mathrm{b}) ; \mathrm{a} \wedge \mathrm{b} \neq 0\} & \text { (Overlapping) } \\
\mathrm{D}:=\{(\mathrm{a}, \mathrm{b}) ; \mathrm{a} \wedge \mathrm{b}=0\} & \text { (Disjointness) } \\
\mathrm{CO}:=\{(\mathrm{a}, \mathrm{b}) ; \mathrm{a} \vee \mathrm{b} \neq 1\} & (\text { Co-Overlapping, Underlapping })^{13} \\
\mathrm{CD}:=\{(\mathrm{a}, \mathrm{b}) ; \mathrm{a} \vee \mathrm{b}=1\} & \text { (Co-Disjointness) } \\
\mathrm{H}:=\left\{\left(\mathrm{a}, \mathrm{a}^{*}\right) ; \mathrm{a} \in \mathrm{H}\right\} & \text { (Heyting complement) } \\
\mathrm{H}^{\circ}:=\left\{\left(\mathrm{a}^{*}, \mathrm{a}\right) ; \mathrm{a} \in \mathrm{H}\right\} & \text { (Converse Heyting complement) } \\
\mathrm{NTP}:=\{(\mathrm{a}, \mathrm{c}) ; \mathrm{a}<<\mathrm{c}\} & \text { (Non-tangential part) }
\end{array}
$$

Evidently, some of these relations can be defined in terms of others by using the above mentioned Boolean operators and the conversion defined on REL(H) e.g.:

$$
\begin{aligned}
& \mathrm{P}=\mathrm{PP} \cup \mathrm{ID} . \\
& \mathrm{D}=\mathrm{CO} . \\
& \mathrm{D}=\mathrm{D}^{\circ}, \mathrm{O}=\mathrm{O}^{\circ}, \mathrm{CD}=\mathrm{CD}^{\circ} \text {, and } \mathrm{CO}=\mathrm{CO}^{\circ} .
\end{aligned}
$$

More interesting than these purely set-theoretical elucidations of spatial relations are those that involve relational products:

(7.3) Proposition. Let $(\mathrm{H}, \leq)$ be a Heyting algebra. Then the following holds:

$$
\begin{aligned}
& \mathrm{H} \bullet \mathrm{H} \bullet \mathrm{H}=\mathrm{H} . \\
& \mathrm{NTP}=\mathrm{P} \cap(\mathrm{D} \bullet \mathrm{CD}) . \\
& \mathrm{EXT}=\mathrm{D} \cap\left(\mathbf{C}\left(\mathrm{NTP} \bullet \mathrm{H}^{\circ}\right) .\right.
\end{aligned}
$$

Proof. (1) follows directly from $\mathrm{a}^{* * *}=\mathrm{a}^{*}(2.4)(5)$. (2) is just a reformulation of (3.1): The first term P just asserts that aNTPb only holds if a is a part of $b$. Now assume a ((D $\bullet$ CD) $c$. By definition this means that there is $b$ such that $a \mathrm{D} \mathrm{b}$ and $\mathrm{bCD} c$, i.e., $a \wedge b=0$ and $b \vee c=$ 1. In a similar, although more complicated manner the intuitive meaning of (3) may be deciphered. Assume a EXT b, and let consider term a D $\cap(\mathbf{C}(\mathrm{NTP} \bullet \mathrm{H})) \mathrm{b}$. It asserts that $\mathrm{a}$ and $\mathrm{b}$ 
do not overlap and do not satisfy (a NTP b* AND b*Hb). Since a D b, a is a part of b*, by definition (2.1) of the Heyting complement $b^{*}$ of $b$. In sum, then, $a$ is a tangential part of $b^{*}$. Due to (3.9) in the same way one obtains that $b$ is a tangential part of $b^{*}$. In other words, $a$ and $\mathrm{b}$ are in exterior contact.

A comprehensive theory of Heyting and (co-)Heyting spatial concepts will certainly require an in-depth study of the relational algebra of spatial concepts. But even if such an investigation has not yet been carried out, one may safely assert already now that the algebra of spatial relations of Heyting and co-Heyting systems offers a rich field for fruitful further research that refutes the wide-spread opinion that the expressive power of mereology is too weak to deal with genuinely spatial concepts.

Appendix (Proofs of Theorem (4.7) and Theorem (4.9)). Since there does not seem available in the literature a point-free proof of Stone's equation (4.9) I have provided one by myself. It is elementary, but a bit involved in that it uses most of the results on Heyting algebras collected in section 2 .

(4.7) Theorem. Let $\mathrm{C}$ be a Co-Heyting algebra, and $\mathrm{a}, \mathrm{b} \in \mathrm{C}$. Then the following holds:

$$
b d(a \wedge b) \vee b d(a \vee b)=b d(a) \vee b d(b)
$$

Proof. The direction $\leq$ is proved separately for $b d(a \wedge b)$ and $b d(a \vee b)$. By definition one has

$$
\begin{aligned}
\operatorname{bd}(a \wedge b) & =(a \wedge b) \wedge(a \wedge b)+ \\
& =(a \wedge b) \wedge(a+\vee b+) \\
& =(a \wedge b) \wedge a+) \vee(b+\wedge b) \wedge a+) \\
& =(b d(a) \wedge b) \vee(b d(b) \wedge a+)) \leq b d(a) \vee b d(b) .
\end{aligned}
$$

Now consider bd( $a \vee b)$. By definition of the boundary we obtain

$$
\begin{aligned}
\mathrm{bd}(\mathrm{a} \vee \mathrm{b}) & =(a \vee b) \wedge(a \vee b)+ \\
\leq & (a \vee b) \wedge(a+\wedge b+) \\
& =(a \vee b) \wedge(a+\wedge b+) \\
& =(a \wedge a+\wedge b) \vee(b \wedge b+\wedge a+)
\end{aligned}
$$




$$
\leq \mathrm{bd}(\mathrm{a}) \vee \mathrm{bd}(\mathrm{b})
$$

Now let us prove the other half of (6.4), namely, $b d(a) \vee b d(b) \leq b d(a \wedge b) \vee b d(a \vee b)$. Instead of proving it directly, for convenience we prove the dual proposition for Heyting algebras:

$$
\operatorname{cbd}(a) \wedge \operatorname{cbd}(b) \geq \operatorname{cbd}(a \vee b) \wedge \operatorname{cbd}(a \wedge b)
$$

Spelt out in detail this amounts to

$$
\left((a \wedge b) \vee(a \wedge b)^{*}\right) \wedge\left((a \vee b) \vee(a \vee b)^{*}\right) \leq\left(a \vee a^{*}\right) \wedge\left(b \vee b^{*}\right)
$$

Proving this proposition for Heyting algebras saves us a lot of asterisks, since for the proof we have to use the technical results of section 2 formulated for Heyting algebras. So we get:

$$
\begin{aligned}
& \left((a \wedge b) \vee(a \wedge b)^{*}\right) \wedge\left((a \vee b) \vee(a \vee b)^{*}\right) \leq\left(a \vee a^{*}\right) \wedge\left(b \vee b^{*}\right) \\
\Leftrightarrow & {\left[\left((a \wedge b) \wedge\left((a \vee b) \vee(a \vee b)^{*}\right)\right] \vee\left[(a \wedge b)^{*}\right) \wedge\left((a \vee b) \vee(a \vee b)^{*}\right)\right] \leq\left(a \vee a^{*}\right) \wedge\left(b \vee b^{*}\right) }
\end{aligned}
$$

This holds if and only if

and

$$
\left[\left((a \wedge b) \wedge\left((a \vee b) \vee(a \vee b)^{*}\right)\right] \leq\left(a \vee a^{*}\right) \wedge\left(b \vee b^{*}\right)\right.
$$

$$
\left.\left[(a \wedge b)^{*}\right) \wedge\left((a \vee b) \vee(a \vee b)^{*}\right)\right] \leq\left(a \vee a^{*}\right) \wedge\left(b \vee b^{*}\right)
$$

The inequality $(1)$ is true, since clearly $(a \wedge b) \wedge\left((a \vee b) \vee(a \vee b)^{*}\right) \leq(a \wedge b) \leq\left(a \vee a^{*}\right) \wedge(b$ $\left.\vee b^{*}\right)$. So it remains to prove (2). Due to the de Morgan law (2.4)(6) valid for Heyting algebras this is equivalent to

$\left.\left[(a \wedge b)^{*}\right) \wedge\left((a \vee b) \vee\left(a^{*} \wedge b^{*}\right)\right)\right] \leq\left(a \vee a^{*}\right) \wedge\left(b \vee b^{*}\right)$

Due to (2. 4) (3) is equivalent to

$\left.\left[\left[(a \wedge b)^{*}\right) \wedge\left((a \vee b) \vee\left(a^{*} \wedge b^{*}\right)\right)\right] \Rightarrow\left(a \vee a^{*}\right) \wedge\left(b \vee b^{*}\right)\right]=1$

By (2.4)(2) is equivalent to

$\left.\left[\left[(a \wedge b)^{*}\right) \wedge\left((a \vee b) \vee\left(a^{*} \wedge b^{*}\right)\right)\right] \Rightarrow\left(a \vee a^{*}\right)\right]=1$

and

$\left[\left[(a \wedge b)^{*}\right) \wedge\left((a \vee b) \vee\left(a^{*} \wedge b^{*}\right)\right)\right] \Rightarrow\left(\left(b \vee b^{*}\right)\right]=1$ 
Both equations are symmetric in a and b. Hence it is sufficient to prove one, say (5a). By applying the distributivity law, we obtain that (5a) is equivalent to

$$
\left.\left[\left[(a \wedge b)^{*}\right) \wedge((a \vee b)] \vee\left[\left[(a \wedge b)^{*}\right) \wedge\left(a^{*} \wedge b^{*}\right)\right)\right] \Rightarrow\left(a \vee a^{*}\right)\right]=1
$$

Applying once again (2.4)(1) this is equivalent

$$
\left.\left[\left[(a \wedge b)^{*}\right) \wedge((a \vee b)] \vee\left[\left[(a \wedge b)^{*}\right) \wedge\left(a^{*} \wedge b^{*}\right)\right)\right] \leq\left(a \vee a^{*}\right)\right]
$$

But clearly $\left.\left.\left[\left[(a \wedge b)^{*}\right) \wedge\left(a^{*} \wedge b^{*}\right)\right)\right] \leq\left(a \vee a^{*}\right)\right]$. Hence there is only left to prove

$$
\left[\left[(a \wedge b)^{*}\right) \wedge((a \vee b)] \leq\left(a \vee a^{*}\right)\right]
$$

Applying distributivity to the left side we obtain that (8) is equivalent to

$\left.\left.\left.\left.\left[(a \wedge b)^{*}\right) \wedge a\right) \vee(a \wedge b)^{*}\right) \wedge b\right)\right] \leq\left(a \vee a^{*}\right)$

Obviously $\left.\left[(a \wedge b)^{*}\right) \wedge a\right) \leq\left(a \vee a^{*}\right)$, and so we are left with $\left.\left.\left.(a \wedge b)^{*}\right) \wedge b\right)\right] \leq\left(a \vee a^{*}\right)$. Clearly this is implied by proving $\left.\left.\left.(a \wedge b)^{*}\right) \wedge b\right)\right] \leq a^{*}$. By the definition of $\Rightarrow$ this holds if and only if $(a \wedge b)^{*} \leq\left(b \Rightarrow a^{*}\right)$. Taking into account that $a^{*}$ is just $(a \Rightarrow 0)$ one has to prove that $(\mathrm{a} \wedge \mathrm{b}) \Rightarrow 0 \leq(\mathrm{b} \Rightarrow(\mathrm{a} \Rightarrow 0)$, since $(\mathrm{b} \Rightarrow(\mathrm{a} \Rightarrow 0)=(\mathrm{a} \wedge \mathrm{b}) \Rightarrow 0$. But this true by (Borceux 3, Proposition 1.2.15, p. 13). This clinches the proof.

Corrollary. Let $(X, O X)$ be a topological space, and $a, b \in O X$. Then

$$
\mathrm{bd}(\mathrm{a} \cap \mathrm{b}) \cup \mathrm{bd}(\mathrm{a} \cup \mathrm{b})=\mathrm{bd}(\mathrm{a}) \cup \mathrm{bd}(\mathrm{b})
$$

Proof: Apply (4.7) to the closed sets $\mathbf{C a}$ and $\mathbf{C b}$ and observe that $\mathrm{bd}(\mathrm{a})=\mathrm{bd}(\mathbf{C a})$ and $\mathrm{bd}(\mathrm{b})=$ $\operatorname{bd}(\mathbf{C b})$

(4.9) Theorem (A.H. Stone). Let (X, OX) be a topological space and and $a, b \in P X$. Then the following formula holds:

$$
\mathrm{bd}(\mathrm{a} \cap \mathrm{b}) \cup(\mathrm{bd}(\mathrm{a} \cup \mathrm{b})) \cup(\mathrm{bd}(\mathrm{a}) \cap \mathrm{bd}(\mathrm{b}))=\mathrm{bd}(\mathrm{a}) \cup \mathrm{bd}(\mathrm{b})
$$

Proof. We first prove the inequality $\subseteq$. This is done separately for each of the three components of the left side. Trivially, one has $(\mathrm{bd}(\mathrm{a}) \cap \mathrm{bd}(\mathrm{b})) \subseteq \mathrm{bd}(\mathrm{a}) \cup \mathrm{bd}(\mathrm{b})$. Hence we have to deal only with the first two factors of the right side. By definition of the boundary operator, for $b d(a \cap b)$ one obtains: 


$$
\begin{aligned}
\mathrm{bd}(\mathrm{a} \cap \mathrm{b}) & =\operatorname{cl}(\mathrm{a} \cap \mathrm{b}) \cap(\operatorname{cl}(\mathbf{C}(\mathrm{a} \cap \mathrm{b})) \subseteq(\operatorname{cl}(\mathrm{a}) \cap \operatorname{cl}(\mathrm{b})) \cap(\operatorname{cl}(\mathbf{C a}) \cup \operatorname{cl}(\mathbf{C b})) \\
& =(\operatorname{cl}(\mathrm{a}) \cap \operatorname{cl}(\mathrm{b}) \cap \operatorname{cl}(\mathbf{C a})) \cup(\operatorname{cl}(\mathrm{a})) \cap \operatorname{cl}(\mathrm{b}) \cap(\mathrm{cl}(\mathbf{C b})) \\
& =(\mathrm{bd}(\mathrm{a}) \cap \operatorname{cl}(\mathrm{b})) \cup(\mathrm{bd}(\mathrm{b}) \cap(\operatorname{cl}(\mathrm{a})) \\
& \subseteq(\mathrm{bd}(\mathrm{a}) \cup \mathrm{bd}(\mathrm{b}))
\end{aligned}
$$

To prove the inclusion of $b d(a \cup b)$ one argues in a similar manner:

$$
\begin{aligned}
\mathrm{bd}(\mathrm{a} \cup \mathrm{b}) & =\operatorname{cl}(\mathrm{a} \cup \mathrm{b}) \cap \operatorname{cl}(\mathbf{C}(\mathrm{a} \cup \mathrm{b})) \\
& =(\operatorname{cl}(\mathrm{a}) \cup \operatorname{cl}(\mathrm{b})) \cap \operatorname{cl}(\mathbf{C a} \cap \mathbf{C b})) \\
& \subseteq(\operatorname{cl}(\mathrm{a}) \cup \operatorname{cl}(\mathrm{b})) \cap(\operatorname{cl}(\mathbf{C a})) \cap \operatorname{cl}(\mathbf{C b})) \\
& =(\mathrm{bd}(\mathrm{a}) \cap \operatorname{cl}(\mathbf{C b})) \cup(\mathrm{bd}(\mathrm{b}) \cap(\operatorname{cl}(\mathbf{C a})) \\
& \subseteq \mathrm{bd}(\mathrm{a}) \cup \mathrm{bd}(\mathrm{b}) .
\end{aligned}
$$

This proves the first half of the equation. To prove the opposite direction, first note that the equation is symmetric in a and $b$, hence it is sufficient to prove

$$
\mathrm{bd}(\mathrm{a}) \subseteq \mathrm{bd}(\mathrm{a} \cap \mathrm{b}) \cup(\mathrm{bd}(\mathrm{a} \cap \mathrm{b})) \cup(\mathrm{bd}(\mathrm{a}) \cap \mathrm{bd}(\mathrm{b}))
$$

Assume $\alpha \in \operatorname{bd}(a)$. Then $\alpha \in \operatorname{int}(b)$ or $\alpha \in$ bd(b) or $\alpha \in \operatorname{int}(\mathbf{C b})$ (cf. Christensen and Voxman (1.E.16), p. 29) This trichotomy is strict and exhaustive, i.e. $\operatorname{int}(b) \cup \operatorname{bd}(b) \cup \operatorname{int}(\mathbf{C b})=X$ and its factors are disjoint.

Assume $\alpha \in \operatorname{int}(\mathrm{b})$. Then there is an open neighborhood $\mathrm{U}(\alpha) \subseteq$ b. By definition of a boundary point every open neighborhood $\mathrm{V}(\alpha)$ intersects nontrivially both with a and $\mathbf{C a}$. We are going to show that in this case $\alpha \in \mathrm{bd}(\mathrm{a} \cap \mathrm{b})$.

$$
\begin{array}{rlrl}
\operatorname{bd}(\mathrm{a} \cap \mathrm{b})= & & \operatorname{cl}((\mathrm{a} \cap \mathrm{b}) \cap \operatorname{cl}(\mathbf{C}(\mathrm{a} \cap \mathrm{b})) . \\
= & & \operatorname{cl}((\mathrm{a} \cap \mathrm{b}) \cap \operatorname{cl}(\mathbf{C a} \cup \mathbf{C b}) . \\
= & (\operatorname{cl}((\mathrm{a} \cap \mathrm{b}) \cap \operatorname{cl}(\mathbf{C a})) \cup(\operatorname{cl}((\mathrm{a} \cap \mathrm{b}) \cap \operatorname{cl}(\mathbf{C b})) .
\end{array}
$$

Since $\alpha \in \operatorname{bd}(a)$ and $b d(a)=b d(\mathbf{C a})$ clearly $\alpha \in \operatorname{cl}(\mathbf{C a})$. Hence it is sufficient to show that $\alpha$ $\in \operatorname{cl}((a \cap b)$. By definition of $c l$ this is the case if and only if every open neighborhood $V(\alpha)$ of $\alpha$ has a non-empty intersection with ( $\mathrm{a} \cap \mathrm{b})$. Since $\alpha \in \operatorname{int}(\mathrm{b})$ one may assume $\mathrm{V}(\alpha) \subseteq \mathrm{b}$. 
Since $V(\alpha) \cap a \neq \varnothing$ since $\alpha \in$ bd(a) we obtain $V(\alpha) \cap a \cap b \neq \varnothing$, i.e. $\alpha$ is an element of $\operatorname{cl}(a$ $\cap b)$.

Now assume $\alpha \in \operatorname{int}(\mathbf{C b})$. We are going to show that this entails $\alpha \in \operatorname{bd}(\mathrm{a} \cup \mathrm{b})$. By definition of bd we get

$$
\begin{array}{rlrl}
\operatorname{bd}(\mathrm{a} \cup \mathrm{b}) & = & & \operatorname{cl}(\mathrm{a} \cup \mathrm{b}) \cap \operatorname{cl}(\mathbf{C}(\mathrm{a} \cup \mathrm{b})) \\
& = & (\operatorname{cl}(\mathrm{a}) \cup \operatorname{cl}(\mathrm{b})) \cap \operatorname{cl}(\mathbf{C a} \cap \mathbf{C b})) \\
& = & (\operatorname{cl}(\mathrm{a}) \cap \operatorname{cl}(\mathbf{C a} \cap \mathbf{C b})) \cup(\operatorname{cl}(\mathrm{b})) \cap \operatorname{cl}(\mathbf{C a} \cap \mathbf{C b}))
\end{array}
$$

Since $\alpha \in \operatorname{bd}(a)$ clearly $\alpha \in \operatorname{cl}(a)$. Hence in order to show that $\alpha \in \operatorname{bd}(a \cup b)$ it is sufficient to prove that $\alpha \in \operatorname{cl}(\mathbf{C a} \cap \mathbf{C b}))$. This is true if and only if every open neighborhood $\mathrm{V}(\alpha)$ of $\alpha$ has a non-empty intersection with $\mathbf{C a} \cap \mathbf{C b}$. Since $\alpha \in \operatorname{int}(\mathbf{C b})$ we can assume $\mathrm{V}(\alpha) \subseteq$ b. By assumption $\alpha \in \mathrm{bd}(\mathrm{a})$ and therefore $\mathrm{V}(\alpha) \subseteq \mathrm{b}$. $\mathrm{V}(\alpha) \cap \mathrm{a}=\mathrm{V}(\alpha) \cap \mathrm{a} \cap \mathrm{b} \neq \varnothing$. Hence in this case one has $\alpha \in \mathrm{bd}(\mathrm{a} \cup \mathrm{b})$ and therefore

$$
\mathrm{bd}(\mathrm{a}) \subseteq \mathrm{bd}(\mathrm{a} \cap \mathrm{b}) \cup(\mathrm{bd}(\mathrm{a} \cap \mathrm{b})) \cup(\mathrm{bd}(\mathrm{a}) \cap \mathrm{bd}(\mathrm{b}))
$$

The analogous inclusion for bd(b) is proved in exactly the same way. This clinches the proof of Stone's theorem.

Stone's theorem holds for every (Boolean) closure algebra $(B, \leq, c)$ not only for those that arise from topological spaces $(\mathrm{X}, \mathrm{OX})$. Recall that a closure algebra $(\mathrm{B}, \leq, \mathrm{c})$ is a Boolean algebra $(\mathrm{B}, \leq)$ endowed with an operator $\mathrm{c}$ on $\mathrm{B}$ that satisfies the algebraic version of the four Kuratowski axioms (cf. Kuratowski and Mostowski (1976, §8, 27), McKinsey and Tarski $(1944,145 / 146)$. Clearly, every topological space (X, OX) gives rise to a closure algebra (PX, $\subseteq$, cl) and vice versa. According to Theorem (2.6) of McKinsey and Tarski $(1944,150)$ every closure algebra $(\mathrm{B}, \leq, \mathrm{c})$ is isomorphic to a subalgebra of a closure algebra of $(\mathrm{PX}, \subseteq, \leq)$ of a topological space $(\mathrm{X}, \mathrm{OX})$. Thus, by embedding of $(\mathrm{B}, \leq, \mathrm{c})$ into the closure algebra (PX, $\subseteq$, $\leq$ ) of a topological space one obtains that Stone's equation also holds for the abstract closure algebra $(\mathrm{B}, \leq, \mathrm{c})$.

\section{Notes:}

\footnotetext{
${ }^{1}$ It goes without saying that this is not to assert that the hybrid accounts of Boolean mereology cum topology should be simply dismissed. I only contend that these „mereotopological“
} 
accounts are not the only possible ones. There are other frameworks for spatial reasoning that deserve to be investigated as well. The Heyting approach sketched in this paper has the advantage of greater conceptual austerity and homogeneity. There are some good metaphysical reasons to believe that the concept a Boolean framework is committed to some unrealistically strong assumptions. For instance the concept of a strict Boolean complement is problematic. After all, if a part of Boolean mereological system represents a physical object what is its Boolean complement to represent? Hence, formulating a mereological framework that is based on weaker assumptions may help uncover the essence of mereological relations as it shows up in the roles they have in spatial reasoning.

In contrast to the existing mereotopological accounts, which may be characterized as conceptual hybrids that combine concepts from two distinct areas, the Heyting account is based on a homogeneous conceptual basis. More precisely, from the perspective of the present paper the Heyting approach provides a convenient general framework for spatial reasoning while the restriction to Boolean systems is seem as a specialization for which the spatial aspects become invisible. Reintroducing spatiality later by adding some allegedly non-mereological notions such as a contact relation or an interior parthood relation appears as an unnecessarily complicated way to come to terms with the conceptual problems posed by spatial reasoning.

${ }^{2}$ A typical example of such an algebra is the Boolean algebra of regular open sets $\mathrm{O} * \mathrm{E}$ of the Euclidean space $\mathrm{E}$.

NB: „Disconnectedness“ is not to be confused with „unrelated to topology“. On the contrary, Stone's famous representation theorem has established a deep connection between topology and the theory of Boolean algebras (cf. Stone (1936), Johnstone (1982). But for the modest purposes of the present paper we need not go into this mathematically more advanced territory.

As will be explained in section 5, the Heyting algebra $\mathrm{H}$ has to be continuous. The crucial point is that "continuity“ in the sense as it is used here, can be defined in purely mereological (lattice-theoretical) terms.

${ }^{4}$ (3.1) especially applies to Heyting algebras. In this case one may take as b in (3.1) the Heyting complement $\mathrm{a}^{*}$ if $\mathrm{a}<<\mathrm{c}$ obtains at all.

${ }^{5}$ For Boolean mereological systems $(\mathrm{B}, \leq)$ the contact relation defined by (3.9) and the relation of overlapping coincide. On the other hand, the mereotopological contact relations for Boolean algebras $(\mathrm{B}, \leq)$ endowed with an extra topological contact relation are usually extensional, i.e. from $\{\mathrm{x} ; \mathrm{xCa}\}=$ $\{\mathrm{x} ; \mathrm{xCb}\}$ it follows $\mathrm{a}=\mathrm{b}$, for $\mathrm{a}, \mathrm{b} \in \mathrm{B}$. Elementary examples show that Heyting contact relations are not extensional.

${ }^{6}$ The dual Heyting concept corresponding to the co-Heyting concept of boundary bd may be dubbed „co-boundary" defined by $\operatorname{cbd}(\mathrm{a}):=\mathrm{a} \vee \mathrm{a}^{*}$. As it seems, up to now, this concept has not yet found many useful applications. But it can be used to characterize Boolean algebras as those Heyting algebras all of whose elements have a trivial coboundary, i.e. for all of them a $\vee a^{*}=1$ (cf. 2.5). In other words, the "Heytingness“ of Heyting algebras resides in the non-triviality of the coboundary cbd. Dually to (2.5) one obtains that a co-Heyting algebra B is a Boolean algebra if and only if $\mathrm{a}++=\mathrm{a}$ and, equivalently, $\mathrm{a} \wedge \mathrm{a}+=0$. In other words, a co-Heyting algebra is Boolean if and only if its elements have trivial boundaries. This is an other evidence that Boolean algebras are unsuitable to deal for with boundaries and other spatial concepts.

${ }^{7}$ The common-sense intuition of thinness for boundaries is not fully captured by the topological concept of boundary. There are many sets whose boundary is quite „thick“. For instance, the boundary $\operatorname{bd}(\mathbf{Q})$ of the rational numbers $\mathbf{Q}$ in the real number $\mathbf{R}$ is "thick“, namely, bd( $\mathbf{Q})=\mathbf{R}$. If a boundary concept bd yields thick boundaries for some kind of objects, it does not seem appropriate for them, or, formulated the other way round, these objects appear to be pathological from the perspective of the chosen concept of boundary.

The dual concept of „thin“ in the framework of Heyting algebras is „dense“ defined by „a is dense if and only if $a^{* *}=1$. Corresponding to (4.2) we obtain that coboundaries a $\vee a^{*}$ are dense in the sense that $\left(\mathrm{a} \vee \mathrm{a}^{*}\right)^{* *}=1$. 
${ }^{8}$ The many logical inaccuracies that can be found in the axiomatizations of mereology by these authors, in particular with respect to the relations between the various types of mereological systems, have been extensively discussed by Pontow and others (cf. Pontow (2004)). These criticisms are not pertinent for the purposes of the present paper, since what is at issue here are certain difficulties concerning topology. The main aim of this paper is not to critizise the extant proposals as mathematically erroneous but to make some novel, more plausible proposals.

${ }^{9}$ It is indeed possible to express the Kuratowski axioms for the closure operator cl purely in terms of the boundary operator bd, but the resulting axioms are rather different from those that Casati and Varzi have proposed (cf. (4.10)).

${ }^{10}$ Note that a frame map need not preserve the Heyting complement, it may happen that $f\left(a^{*}\right) \neq f(a)^{*}$. Indeed, a functional point $\mathrm{OX}---\mathrm{f}_{\mathrm{y}}----->>2, \mathrm{y} \in \mathrm{X}$, usually does not preserve the Heyting complement, since for $a \in O X$ and $y \in b d(a)$ one obtains $f_{y}(a)=f_{y}\left(a^{*}\right)=0$ and therefore $0=f_{y}\left(a^{*}\right) \neq f_{y}(a)^{*}=1$. The existence of non-trivial (co)boundaries forces functional points to be frame homomorphisms instead of *-preserving Heyting homomorphisms. Only if $\mathrm{H}$ happens to be Boolean, its functional points are Boolean homomorphisms that respect Boolean complements.

${ }^{11}$ Originally, the models of GEM - as allegedly being those of ,classical mereology“ - were claimed to be complete Boolean algebras with the bottom element removed (Casati and Varzi 1999, 46). Later, it was shown by Pontow and others that this is strictly speaking not true (cf. Pontow (2004) and Hovda (2009)). For the purposes of the present paper it is not necessary to discuss this issue. Rather, I assume that the axiom systems of Casati, Varzi, Smith, and others, intended to describe the models of classical extensional mereology as complete Boolean algebras (with the bottom element deleted) indeed achieve this goal. The fact that their axioms fail to achieve this, is of no concern to us.

12 There is, albeit not under this name, an elaborated theory of the mereology of Heyting algebras conceived of as objects of the category of locales, see Borceux (1994, vol 3, chapter 1). In category theory that, what is in mereology used to be called a part of an object, is called a ,subobject" of that object. Thereby, every category comes along with its own category-specific mereology, namely the theory of the subobjects of the objects of the category (cf. Lawvere and Rosebrugh 2003, chapter 2).

${ }^{13}$ If $(\mathrm{H}, \leq)$ is replaced by its dual $\left(\mathrm{H}^{\mathrm{opp}}, \leq^{\mathrm{opp}}\right)$ the relations $\mathrm{CO}$ and $\mathrm{CD}$ on $(\mathrm{H}, \leq)$ become $\mathrm{O}^{\text {opp }}$ and $\mathrm{D}^{\text {opp }}$ on $\left(\mathrm{H}^{\text {opp }}, \leq^{\text {opp }}\right)$.

\section{$\underline{\text { References. }}$}

Aiello M, Pratt-Hartmann IE, van Benthem JFAK (eds) (2007) Handbook of Spatial Logics. Springer.

Awodey S (2010) Category Theory. Second edition, Oxford University Press, Oxford.

Borceux F (1994) Handbook of Categorical Algebra 3, Categories of Sheaves. Cambridge University Press, Cambridge.

Casati R, Varzi AC, (1999) Parts and Places, The Structures of Spatial Representation, MIT Press, Cambridge/Massachusetts.

Cohen AG, Varzi AC (2003) Mereotopological Connection. Journal of Philosophical Logic 32: $357-390$

Davey BA, Priestley HA, (1990) Introduction to Lattices and Order. Cambridge University Press, Cambridge. 
Düntsch I, Wang H, McCloskey S (1999) Relation Algebras in Qualitative Spatial Reasoning. Fundamenta Informaticae 39: 229 - 248

Düntsch I, Schmidt G, Winter M (2001) A Necessary Relation Algebra for Mereotopology. Studia Logica 69: 381- 409

Gierz G, Hofmann KH, Keimel K, Lawson JD, Mislove M, Scott DS (2003) Continuous Domains and Lattices. Cambridge University Press, Cambridge.

Grätzer G (1998) General Lattice Theory, Second Edition. Birkhäuser, Basel.

Hovda P (2009) What is Classical Mereology? Journal of Philosophical Logic 38(1): 55 - 82

Kuratowski K, Mostowski A (1976) Set Theory. With an Introduction to Descriptive Set Theory. North-Holland, Amsterdam, New York, Oxford.

Lawvere, FW (1986) Introduction, in FW Lawvere and SH Schanuel (ed), Categories in Continuum Physics, Lecture Notes in Mathematics 1174. Springer. Berlin, Heidelberg, $1-16$

Lawvere FW, Rosebrugh R (2003) Sets for Mathematicians. Cambridge University Press, Cambridge.

McKinsey JCC, Tarski A (1944) The Algebra of Topology. Annals of Mathematics 45(1): $141-191$

Mac Lane S, Moerdijk I (1992) Sheaves in Geometry and Logic, A First Introduction to Topos Theory. Springer.

Pontow C (2004) A note on the axiomatics of theories in parthood. Data \& Knowledge Engineering 50: $195-213$

Reyes G, Zolfaghari H (1996) Bi-Heyting Algebras, Toposes and Modalities. Journal of Philosophical Logic 25(1): 25 - 43

Smith B (1996) Mereotopology: a theory of parts and boundaries. Data \& Knowledge Engineering 20: 287 - 303

Smith B, Varzi AC (2000) Fiat and Bona Fide Boundaries. Philosophy and Phenomenological Research 60: $401-420$

Stone M (1936) The theory of representations for Boolean algebras. Transactions of the American Mathematical Society 40: $37-111$

Tarski A (1941) On the calculus of relations. Journal of Symbolic Logic 6: 73 - 89

Varzi, AC (1996) Parts, wholes, and part-whole relations: the prospects of mereotopology.

Data \& Knowledge Engineering 20: 259 - 289 
Varzi AC. (2007) Spatial Reasoning and Ontology: Parts, Wholes, and Locations, in M. Aiello M, Pratt-Hartmann IE, van Benthem JFAK (eds), Handbook of Spatial Logic, Springer, Berlin, Heidelberg, New York, pp945 - 1038 